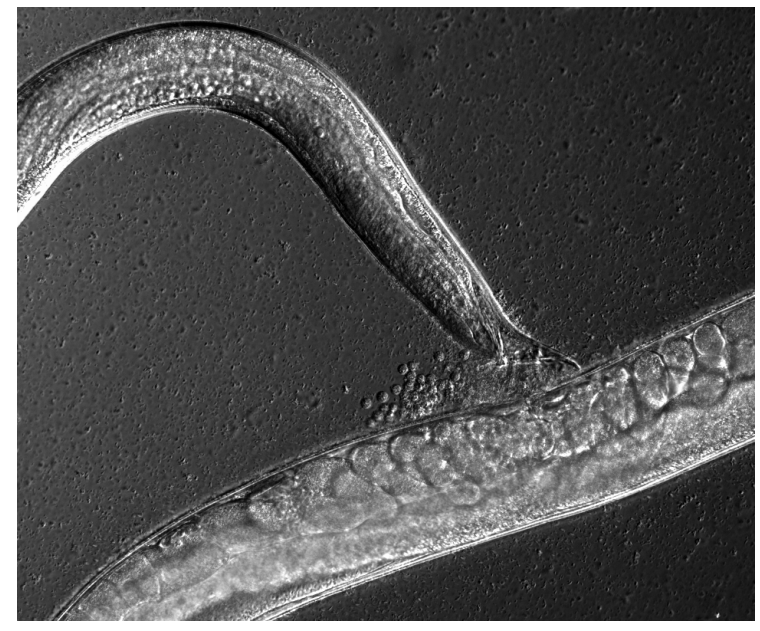

\title{
Evolution of sperm competition: Natural variation and genetic determinants of Caenorhabditis elegans sperm size
}

\author{
${ }^{1}$ Université Côte d'Azur, CNRS, Inserm, IBV, Nice, France \\ ${ }^{2}$ Instituto Gulbenkian de Ciencia, Oeiras, Portugal \\ ${ }^{3}$ Department of Molecular Biosciences, Northwestern University, Evanston, IL USA \\ ${ }^{4}$ School of Biological Sciences, Georgia Institute of Technology, Atlanta, GA USA \\ * these authors contributed equally \\ \# corresponding author
}

\begin{abstract}
Sperm morphology is critical for sperm competition and thus for reproductive fitness. In the male-hermaphrodite nematode Caenorhabditis elegans, sperm size is a key feature of sperm competitive ability. Yet despite extensive research, the molecular mechanisms regulating $C$. elegans sperm size and the genetic basis underlying its natural variation remain unknown. Examining 97 genetically distinct $C$. elegans strains, we observe significant heritable variation in male sperm size but genome-wide association mapping did not yield any QTL (Quantitative Trait Loci). While we confirm larger male sperm to consistently outcompete smaller hermaphrodite sperm, we find natural variation in male sperm size to poorly predict male fertility and competitive ability. In addition, although hermaphrodite sperm size also shows significant natural variation, male and hermaphrodite sperm size do not correlate, implying a sex-specific genetic regulation of sperm size. To elucidate the molecular basis of intraspecific sperm size variation, we focused on recently diverged laboratory strains, which evolved extreme sperm size differences. Using mutants and quantitative complementation tests, we demonstrate that variation in the gene nurf-1 - previously shown to underlie the evolution of improved hermaphrodite reproduction - also explains the evolution of reduced male sperm size. This result illustrates how adaptive changes in $C$. elegans hermaphrodite function can cause the deterioration of a male-specific fitness trait due to a sexually antagonistic variant, representing an example of intralocus sexual conflict with resolution at the molecular level. Our results further provide first insights into the genetic determinants of C. elegans sperm size, pointing at an involvement of the NURF chromatin remodelling complex.
\end{abstract}

Key Words: Androdieocy, Caenorhabditis, intralocus sexual conflict, male function, nurf-1, NURF chromatin remodelling complex, sperm competition, sperm dimorphism

Running Title: Genetic basis of C. elegans sperm size variation 


\section{INTRODUCTION}

Sexual selection in the form of sperm competition is hypothesized to be responsible for rapid evolutionary divergence of sperm traits (Birkhead et al. 2009; Birkhead and Moller 1998; Pitnick et al. 2009; Ramm et al. 2014; Smith 1984; Snook 2005). In particular, sperm morphology may show extreme variation in size and shape among species, and is often associated with variation in competitive ability and thus male reproductive success. Furthermore, disparities of specific sperm traits, such as cell size or flagellum length, is not only common among species, but also within species (Pitnick et al. 2009). Numerous studies focusing on intraspecific variation, through comparison of sperm traits across populations of divergent individuals or by using artificial selection on sperm traits, have uncovered extensive levels of heritable variation in diverse sperm characteristics (Simmons and Moore 2009; Pitnick et al. 2009; Ward 1998; Joly et al. 2004; Morrow and Gage 2001a). Despite such studies on diverse invertebrate and vertebrate taxa, the quantitative and molecular genetic architecture of sperm traits associated with competitive ability remain largely undescribed. Therefore, although the developmental genetics of spermatogenesis and specific sperm traits have been elucidated in great detail from model organisms, such as Drosophila melanogaster (Demarco et al. 2014) or Caenorhabditis elegans (Ellis and Stanfield 2014), it is largely unknown whether uncovered genes are also a substrate for evolution to affect intraspecific variation in sperm characters relevant for male competitiveness.

Here we aimed to quantify and characterize intraspecific genetic variation of a well defined sperm trait (cell size) known directly to affect sperm competitive ability in the androdioecious (male-hermaphrodite) nematode C. elegans. Nematodes of the Caenorhabditis genus have become useful organisms to study sperm competition because it was demonstrated that the size of amoeboid Caenorhabditis sperm is a key factor for sperm competitive ability (Ward and Carrel 1979; LaMunyon and Ward 1995, 1998, 1999, 2002; Palopoli et al. 2015; Ting et al. 2014). In C. elegans, both males and hermaphrodites produce sperm, so that hermaphrodites can either self-fertilize or outcross with males; however, hermaphrodites cannot inseminate each other. C. elegans shows pronounced sperm size dimorphism: male sperm is larger and consistently outcompetes smaller hermaphrodite sperm when both types of sperm are present in the hermaphrodite reproductive tract (Ward and Carrel 1979; LaMunyon and Ward 1995). Male sperm size is further critical when multiple males compete for fertilization, with larger sperm outcompeting smaller sperm (LaMunyon and Ward 1998; Murray et al. 2011). In addition, increased levels of male-male competition in experimental contexts lead to the evolution of larger male sperm size (LaMunyon and Ward 2002; Palopoli et al. 2015), consistent with the relevance of sperm size for male competitive ability. Moreover, gonochoristic (male-female) Caenorhabditis species - with high levels of malemale competition - exhibit, on average, much larger male sperm than the three androdieocious species, C. briggsae, C. elegans, and C. tropicalis, in which male-male competition is much weaker (LaMunyon and Ward 1999; Vielle et al. 2016).

Although self-fertilization is the predominant mode of $C$. elegans reproduction, with reported rare occurrence of males and outcrossing events in natural populations (Jovelin et al. 2003; Barrière and Félix 2005; Sivasundar and Hey 2005), ample natural variation in diverse male traits exists (Hodgkin and Doniach 1997; Alcorn et al. 2016; Teotonio et al. 2006; Anderson et al. 2010; Noble et al. 2015; Morran et al. 2009; Palopoli et al. 2015; Palopoli et al. 2008), including male sperm size (LaMunyon and Ward 1998; Murray et al. 2011; Vielle et al. 2016). It remains unclear, however, to what degree such variation in male phenotypes reflects variation in male reproductive performance or competitive ability as a result of malehermaphrodite or male-male interactions. Consistent with low rates of outcrossing, a number of male (and hermaphrodite) traits directly linked to mating, such as mate recognition, mating ability, or sex pheromone production are impaired, e.g. relative to related gonochoristic 
species (Sherlekar and Lints 2014; Kleemann and Basolo 2007; Noble et al. 2015; Lipton et al. 2004; Chasnov et al. 2007; Chasnov 2013; Garcia 2014; Thomas et al. 2012; Yin et al. 2018). In contrast, larger male sperm size was shown to clearly associate with increased male competitive ability and reproductive success, and male sperm size varies across C. elegans isolates (Ward and Carrel 1979; LaMunyon and Ward 1995, 1998, 1999, 2002; Palopoli et al. 2015; Murray et al. 2011). In the most comprehensive analysis to date, Murray et al. (2011) showed larger male sperm size to be a highly significant factor explaining natural variation in male fertilization success and male-male competitive ability across seven distinct $C$. elegans wild isolates. Extensive past research on $C$. elegans sperm competition thus indicates that male sperm size is tightly linked to male reproductive performance. Nevertheless, we have only limited knowledge of the extent of natural variation in C. elegans male sperm size, and we often ignore if this variation is adaptive as a result of (sexual) selection. Importantly, it also remains unclear how males and hermaphrodites generate differently sized sperm and to what extent sexual conflict in sperm size determination is resolved given that sperm size optima are strongly divergent between sexes: production of larger sperm will enhance male competitive ability while production of smaller sperm will accelerate hermaphrodite spermatogenesis to reduce developmental time until age of maturity, critical for reproductive fitness (Hodgkin and Barnes 1991; Cutter 2004). Currently, we lack information on how male sperm phenotypes, like other homologous traits shared across sexes, might be influenced by selection on hermaphrodite phenotypes given that hermaphrodite selfing is the predominant mode of reproduction in this species. In particular, we do not know whether malehermaphrodite sperm size shows cross-sexual correlations across genotypes. Furthermore, although ample evidence exists for sperm traits such as length or size, these traits often covary with other traits, such as body size (allometry) or reproductive morphology of females (Pitnick et al. 2009).

Another large gap in our understanding of $C$. elegans sperm competition is the nearcomplete absence of information on the genetic determinants underlying sperm size variation and additional sperm phenotypes relevant for sperm competition. Although the genetic regulation of spermatogenesis has been elucidated in great detail (Chu and Shakes 2013; Ellis and Stanfield 2014; Geldziler et al. 2011; L'Hernault 2006), limited information is available on the molecular genetic control of sperm competitive ability (Hansen et al. 2015) and sperm size determination. No genes specifically affecting $C$. elegans sperm size have so far been identified. Spermatogenesis of $C$. elegans hermaphrodites and males seem essentially identical: meiosis is initiated by the formation of primary spermatocytes, followed by two rapid, mostly symmetrical divisions resulting in four haploid spermatids and an anucleate residual body (Shakes et al. 2009; Vielle et al. 2016; Ward et al. 1981). The cell size of the primary spermatocyte is a key determinant of final spermatid size (Vielle et al. 2016). Specifically, in Caenorhabditis nematodes, primary spermatocyte size differences between species, genotypes within species, and sexes within species strongly correlate with sperm size differences (Vielle et al. 2016). Therefore, sperm size seems to be mostly determined prior to or at the time of primary spermatocyte formation. However, it remains unknown how genetic factors contribute to this process. In addition, it is unclear to what extent the differential presence or activity of such potential genes explain reported differences in sperm size across sexes, genotypes, or species.

Here, we took advantage of a recently established world-wide collection of nearly 100 wild isolates (Andersen et al. 2012; Cook et al. 2017) to characterize the genetic basis of natural variation in $C$. elegans male sperm size. Our specific objectives were (i) to provide a quantitative assessment of natural variation in a presumed male-specific fitness trait in a partially selfing species; (ii) to perform a genome-wide association study to detect potential genetic loci explaining variation in male sperm size; (iii) to examine how observed natural 
variation in male sperm size correlates with variation in male reproductive performance and additional morphological traits of both males and hermaphrodites, including hermaphrodite sperm size; (iv) to characterize the molecular genetic basis of intraspecific variation in $C$. elegans male sperm.

\section{MATERIAL \& METHODS}

\section{Strains and culture conditions}

All strains were maintained at $20^{\circ} \mathrm{C}$ on $2.5 \%$ agar NGM (Nematode Growth Medium) plates seeded with the $E$. coli strain OP50 (Stiernagle 2006). The following strains/genotypes were used in this study: 95 C. elegans wild isolates (Table S1) (Andersen et al. 2012; Cook et al. 2017) and laboratory strains N2, LSJ1, LSJ2, CX12311(kyIR1 V, CB4856>N2; qqIR1 X, CB4856>N2), CX13248 (kyIR84 II, LSJ2>N2), nurf-1(n4295) (MT13649), isw-1(n3294) (MT17795), isw-1(n3297) (MT16012), pyp-1(n4599) IV/nT1 [qIs51] (MT14910), PD4790, and C. plicata (SB355). Hermaphrodites of the mutant fog-2(q71) (strain CB4108) do not produce any self-sperm, i.e. they are effectively females (Schedl and Kimble 1988), which were used for certain mating assays. The strain PD4790 contains an integrated transgene (mls12 [myo-2::GFP, pes-10::GFP, F22B7.9::GFP]) in the N2 reference genetic background, expressing green fluorescent protein (GFP) in the pharynx. Additional information on wild isolates is available from CeNDR (http://elegansvariation.org) (Cook et al. 2017)

\section{Measurements of male and hermaphrodite sperm size}

Males were collected from strain cultures at the L4 stage to be maintained on NGM plates containing only males to measure their spermatid size at stage L4+24h from synchronized and unmated males. Hermaphrodite spermatids were dissected from young virgin adults (at around mid L4+24h), at which stage most individuals contained both spermatids and activated sperm (spermatozoa), the latter of which were excluded from analysis. To measure sperm size, male or hermaphrodite spermatids were released into sperm medium (50mM HEPES $\mathrm{pH} 7.8,50 \mathrm{mM} \mathrm{NaCl}, 25 \mathrm{mM} \mathrm{KCl}, 5 \mathrm{mM} \mathrm{CaCl}_{2}, 1 \mathrm{mM} \mathrm{MgSO}_{4}, 1 \mathrm{mg} / \mathrm{ml} \mathrm{BSA}$ ) by needle dissection (Nelson and Ward 1980). Images of the spermatids were captured using Nomarski optics (60x or $63 x$ objectives). ImageJ software (Rasband, W.S., ImageJ, U. S. National Institutes of Health, Bethesda, Maryland, USA, http://imagej.nih.gov/ij/, 1997-2014) to calculate length and width of each spermatid to obtain measures of cross-sectional area assuming an ellipse shape: $\pi \mathrm{x}($ length/2) $\mathrm{x}$ (width/2) (Vielle et al. 2016).

\section{Male mating ability}

All mating assays (Figures 3 and 4) were performed on mating plates (35mm diameter NGM plates seeded with a spot of $20 \mu \mathrm{l}$ OP50) using unmated males, fog-2 females, or hermaphrodites that had been isolated at the L4 larval stage 24 hours (hermaphrodites and females) or 36 hours (males) prior to the assay. Following established protocols (Murray et al. 2011; Wegewitz et al. 2008), ten virgin fog-2 females were transferred onto a mating plate and allowed to roam for 30 minutes on the bacterial lawn. Next, a single male was added to the plate and allowed to mate for eight hours, after which it was discarded. Females were left on the mating plate for an additional hour and were then picked as single animals onto fresh NGM plates. Females were scored as not fertilized if they did not contain any embryos in the uterus 24 hours later. Offspring production of fertilized females was followed over four consecutive days. 


\section{Male sperm number transferred during single insemination (ejaculate size)}

We quantified the number of sperm transferred during single insemination events (Figures $3 \mathrm{E}$ to 3H). For each strain to be tested (N2, CB4856, LSJ1, JU561, CX11285, EG4946, JU393, and JU782), unmated fog-2 females were individually mated with an excess of 20-30 males, aged 36 hours post-L4 larval stage, to increase the chance of mating. Mating was monitored every few minutes by observation through the stereoscope. When a male was engaged in mating, it was kept under constant surveillance for spicule insertion and visualization of sperm flow from the male vas deferens to the female uterus, until mating was completed. Immediately after the end of mating, the inseminated female was isolated and fixed in icecold methanol and the mated male was removed from the male pool. A new virgin female was then mated with the males. Next, fixed females were washed twice in M9 and mounted in DAPI-containing Vectashield (Vector Laboratories, Inc., Burlingame, CA 94010). Sperm number was counted on images taken at 40x magnification as Z-stacks covering the entire thickness of the gonad using an Olympus BX61 microscope with a CoolSnap HQ2 camera (Poullet et al. 2016; Poullet et al. 2015).

\section{Hermaphrodite-male sperm competition}

We measured variation in competition between hermaphrodite and male sperm by measuring male fertilization success of the eight strains when mated to hermaphrodites of a tester strain (the wild isolate CB4856) (Figure 4A). L4 hermaphrodites of the CB4856 strain were isolated 24 hours prior to mating, and L4 males of the eight strains were isolated 36 hours prior to mating. On the next day, one single CB4856 adult hermaphrodite was mated with an excess of 20-30 males for each strain to be tested and kept under surveillance for single mating as described above. As soon as mating was completed, the male was discarded from the male pool and the hermaphrodite was isolated onto a fresh NGM plate. Offspring (and male) production was scored for three days after the mating assay (i.e. until completion of the reproductive span).

\section{Male-male sperm competition}

We measured second-male sperm precedence of the eight $C$. elegans strains using a tester strain expressing GFP in the pharynx (PD4790), following previously used protocols (Murray et al. 2011) (Figures 4C to 4E). fog-2 females were first mated with PD4970 males, then with males of the eight strains. Males and fog-2 females were isolated at the L4 stage and maintained in isolation for 36 hours prior to mating assays. Each mating plate $(\mathrm{N}=20)$ was established by adding ten fog- 2 females and 20 PD4790 males, which were allowed to mate for 15 hours, so that all females were fertilized (confirmed by the presence of embryos in the uterus). Ten Fertilized fog- 2 females were then randomly allocated to each new mating plate and allowed to mate with 20 males of each of the eight strains examined. Plates were kept under surveillance for single mating as described above. Upon completion of a mating event, both male and female were removed, and offspring production of the female was observed for the next four days. Total offspring were counted using a regular stereoscope and GFPexpressing offspring were counted with a fluorescence stereoscope.

\section{Quantification of hermaphrodite self-sperm number}

We quantified the number of self-sperm in synchronized young adult hermaphrodites, i.e. adults containing one or two embryos in their uterus (Figure 5C). Animals were fixed overnight in ice-cold methanol $\left(-20^{\circ} \mathrm{C}\right)$, washed three times in $1 \mathrm{X}$ PBS containing $0.05 \%$ Tween and mounted in Vectashield (Vector Lab) supplemented with DAPI. Sperm images were acquired from adults containing oocytes to ensure that the sperm to oocyte transition had occurred. Imaging of the anterior spermatheca was performed with an Olympus BX61 
microscope using a $63 \mathrm{X}$ objective with epifluorescence. Z-sections $(1 \mu \mathrm{m})$ of the entire spermatheca were taken and sperm number counted (Cell counter plugin in ImageJ) (Poullet et al. 2016; Poullet et al. 2015)

\section{Body size measurements}

Synchronized populations were used to isolate unmated males in the mid-L4 stage and were scored 24 hours later. Hermaphrodites were scored as early adults when they contained between one and two embryos in the uterus. Animals were then anesthetized in sodium azide on an agar pad and whole-animal images were captured immediately after under Nomarski optics (20X). Body length and width were measured with the ImageJ software and body volume was calculated as that of a cylinder $\left(\pi \times(\text { width } / 2)^{2} \times\right.$ length).

\section{Primary spermatocyte measurements}

Extruded gonads from unmated males at 24 hours post-L4 were obtained by dissection in levamisole-containing M9. Gonads were fixed in 4\% paraformaldehyde for 10 minutes and permeabilized for 5 minutes in $1 \mathrm{X}$ PBS $1 \mathrm{X}$ with $0.1 \%$ Triton X-100. Gonads were next stained for actin with phalloidin (1:500 dilution, Sigma-Aldrich) overnight at $4^{\circ} \mathrm{C}$ in a humidified chamber. Slides were mounted in Vectashield supplemented with DAPI (Vector Lab) and observed under an epifluorescent microscope. Primary spermatocyte area was measured by outlining cell boundaries using ImageJ software (Vielle et al. 2016).

\section{RNAi experiments}

RNAi by bacterial feeding for C. elegans (N2) and C. plicata (SB355) was performed as previously described (Timmons and Fire 1998; Kamath et al. 2003). Briefly, control RNAi (HT115) and nurf-1 clone (provided by the Ahringer lab) were seeded on standard NGM with $50 \mathrm{ug} / \mathrm{ml}$ of ampicillin and $1 \mathrm{mM}$ of IPTG and grow at RT for at least $24 \mathrm{~h}$ before experiment. Worms were fed RNAi and control bacteria from the L1 stage and spermatid size was measured in the early adult stage (L4+24h).

\section{Genome-wide association mapping}

Genome-wide association (GWA) mapping was performed using phenotype data from $97 \mathrm{C}$. elegans isotypes (Table S2). We used the cegwas R package for association mapping (Cook et al. 2017). This package uses the EMMA algorithm for performing association mapping and correcting for population structure (Kang et al. 2008), which is implemented by the GWAS function in the rrBLUP package (Endelmann 2011). Specifically, the GWAS function in the rrBLUP package was called with the following command: $r r B L U P:: G W A S$ (pheno $=$ ph, geno $=y, K=$ kin, $\min \cdot M A F=0.05$, n.core $=1, P 3 D=F A L S E$, plot $=F A L S E)$. The kinship matrix used for association mapping was generated using a whole-genome high-quality single-nucleotide variant (SNV) set from CeNDR release 20160408 (Cook et al. 2016; Evans et al. 2017; Zdraljevic et al. 2017) and the A.mat function from the $r r B L U P$ package. SNVs previously identified using RAD-seq (Andersen et al. 2012) that had at least 5\% minor allele frequency in this strain set were used for performing GWA mappings. Burden test analyses were performed using RVtests (Zhan et al. 2016) and the variable-threshold method (Price et al. 2010). We called SNVs using bcftools (Li 2011) with settings previously described (Zhan et al. 2016; Cook et al. 2017; Cook et al. 2016). We next performed imputation using BEAGLE v4.1 (Cook et al. 2017) with window set to 8000, overlap set to 3000 , and ne set to 17500. Within RVtests, we set the minor allele frequency range from 0.003 to 0.05 for burden testing. 


\section{Statistical analyses}

Statistical tests were performed using R, JMP, or SPSS. Data for parametric tests were transformed where necessary to meet the assumptions of ANOVA procedures (homogeneity of variances and normal distributions of residuals); all size data were log-transformed. For post hoc comparisons, Tukey's honestly significant difference (HSD) procedure was used. For data, where ANOVA assumptions could not be met, we used nonparametric tests (e.g. Kruskal-Wallis).

Broad-sense heritability $\left(H^{2}\right)$ was estimated using the lmer function in the lme4 package (Bates et al. 2015) with the linear mixed model (phenotype $\sim 1+\left(1\right.$ strain). $H^{2}$ was then calculated as the fraction of the total variance explained by the random component (strain) of the mixed model.

\section{Data accessibility}

All raw data are provided in Additional File S1.

\section{RESULTS}

\section{Natural variation in $C$. elegans male sperm size}

We quantified male sperm size variation of a world-wide collection of 97 C. elegans strains (Andersen et al. 2012), including two related laboratory strains (N2 and LSJ1), using measures of spermatid cross-sectional area. Average male sperm size, ranging from $15 \mu \mathrm{m}^{2}$ to $27 \mu \mathrm{m}^{2}$, varied significantly across strains (Figure 1 and Table S2). 90\% of strains exhibited a male sperm size between $20 \mu \mathrm{m}^{2}$ and $25 \mu \mathrm{m}^{2}$, and we detected only two significant outliers: the wild strain JU561 (France) and the laboratory-adapted strain LSJ1 (McGrath et al. 2011) with the smallest male sperm size (Figures 1A and 1B). As found previously for C. elegans and other Caenorhabditis species (Vielle et al. 2016), we also detected high levels of interindividual and intra-individual variation in male sperm size for most strains (Figures 1A and 1C).

Coefficients of variation (CV), i.e. the ratio of the standard deviation to the mean, in sperm characters have been predicted - and shown - to be lower in species or genotypes experiencing higher levels of sperm competition (Fitzpatrick and Baer 2011; Gomendio et al. 2006; Immler et al. 2008; Calhim et al. 2007; Kleven et al. 2008). Therefore, we tested whether $C$. elegans strains with larger male sperm showed reduced variability. However, we did not detect a negative correlation between mean and $\mathrm{CV}$ of within-individual ( $\rho$ Pearson $=0.14, \mathrm{P}=0.17, \mathrm{~N}=97$ ) or between-individual ( $\rho$ Pearson $=-0.09, \mathrm{P}=0.36, \mathrm{~N}=97$ ) male sperm size (Table S2), as expected under such a scenario. 


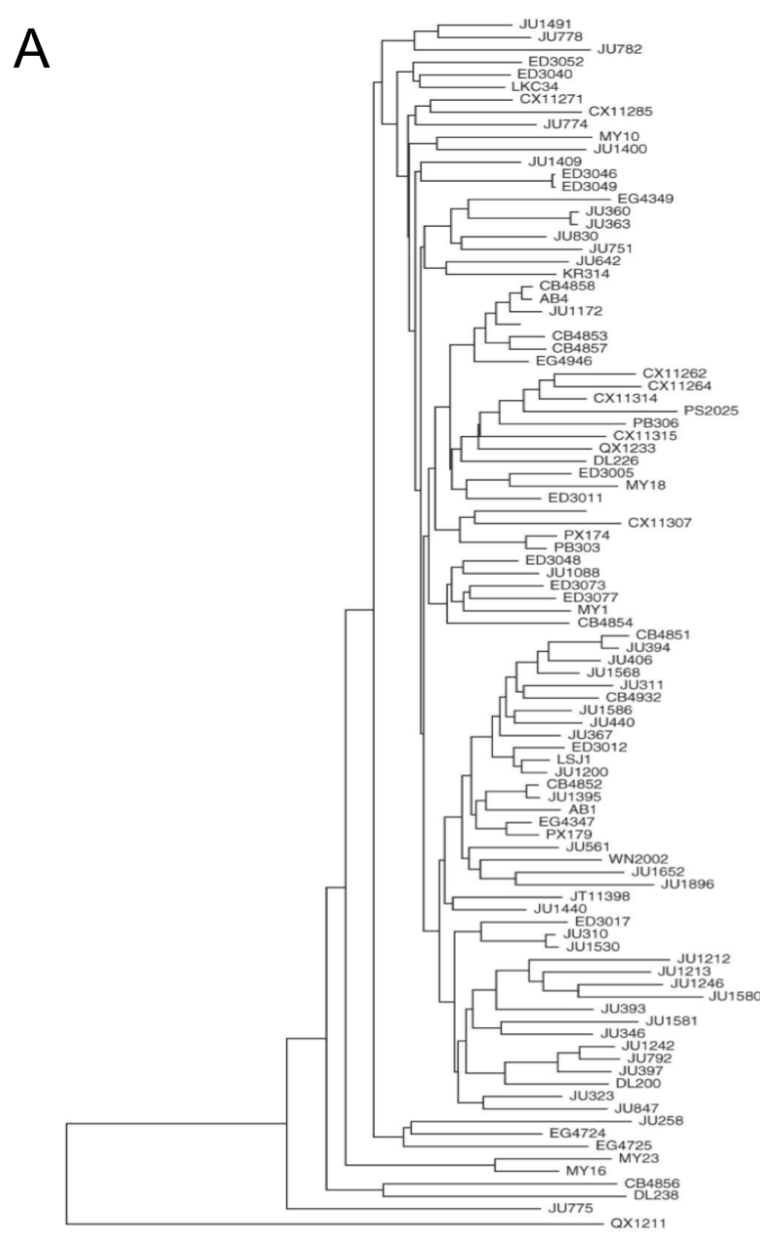

B

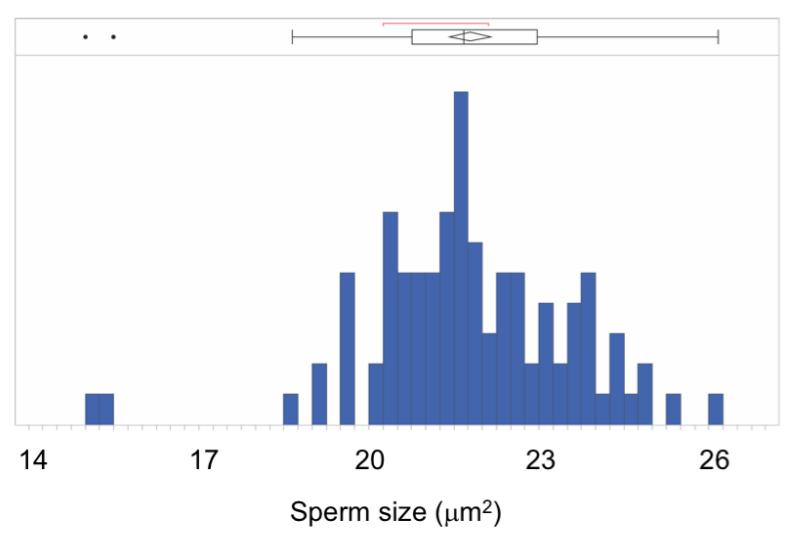

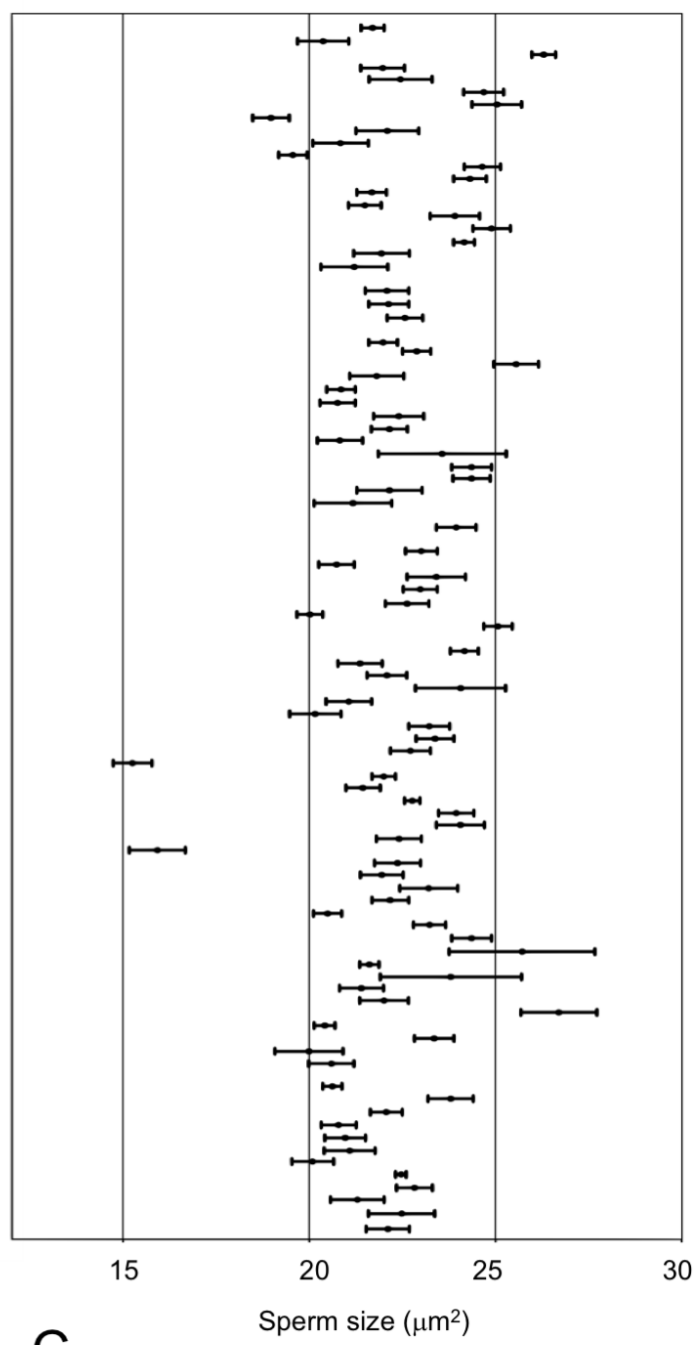

C

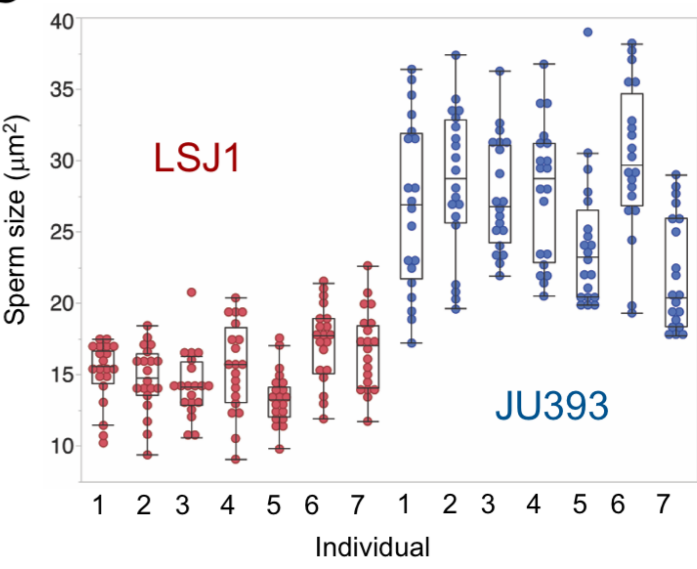

Figure 1. Natural variation in C. elegans male sperm size

(A) Quantification of male spermatid cross-sectional area area (mean \pm sem) in 97 C. elegans strains, arranged with respect to the neighbour-joining tree of Andersen et al. (2012) based on genome-wide SNP data. There is significant genetic (and inter-individual) variation in sperm size (ANOVA, effect strain: $\mathrm{F}_{96,13539}=24.75$, $\mathrm{P}<0.0001$; effect individual(strain): $\mathrm{F}_{580,} 13539=3.08, \mathrm{P}<0.0001$ ). Twenty spermatids from each of seven individuals were measured per strain $(\mathrm{N}=140)$ with the exception of strains JU397 and KR413 for which 20 spermatids from each of six individuals $(\mathrm{N}=120)$ were measured (Table $\mathrm{S} 2)$. (B) Histogram of sperm size across strains (least-squares mean estimates) shows the significant outlier trait values for the two strains (JU561 and LSJ1) with smallest male sperm. (C) Illustration of inter- and intra-individual variation in male sperm size for strains with smallest (LSJ1) versus largest male sperm size (JU393) ( $N=20$ sperm per individual). 


\section{Genome-wide association mapping of male sperm size}

The significant variation in male sperm size enabled the mapping of genomic regions that could underlie this variation using genome-wide association studies, as has been performed successfully for a variety of traits using this C. elegans isolate panel (Andersen et al. 2012; Ashe et al. 2013; Ghosh et al. 2012). Broad-sense heritability was low ( 14\%) for both sperm size cross-sectional area and diameter, and we found no significant genomic regions for these two traits and additional sperm size traits, including CV measurements (Figure 2). This result suggests that many loci could regulate differences in sperm size. Additionally, we used rarevariant based burden testing (Zhan et al. 2016; Bates et al. 2015; Price et al. 2010) to look for association of genes affected by deleterious rare variants with sperm area and diameter. As with marker-based association testing, we did not identify any significant genomic regions (data not shown).

Because specific natural niches could drive mating preferences, we investigated any effect of geography (e.g. latitude/longitude of strain origin) (Table S1, CeNDR: https://www.elegansvariation.org) on average male sperm size but found no such relationship (Spearman rank correlations, all $\mathrm{P}>0.05$ ).

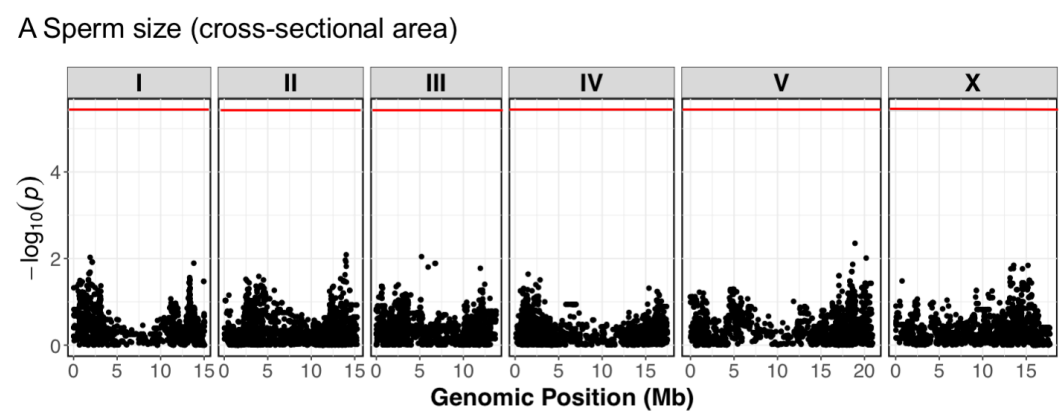

B Sperm size (mean diameter)

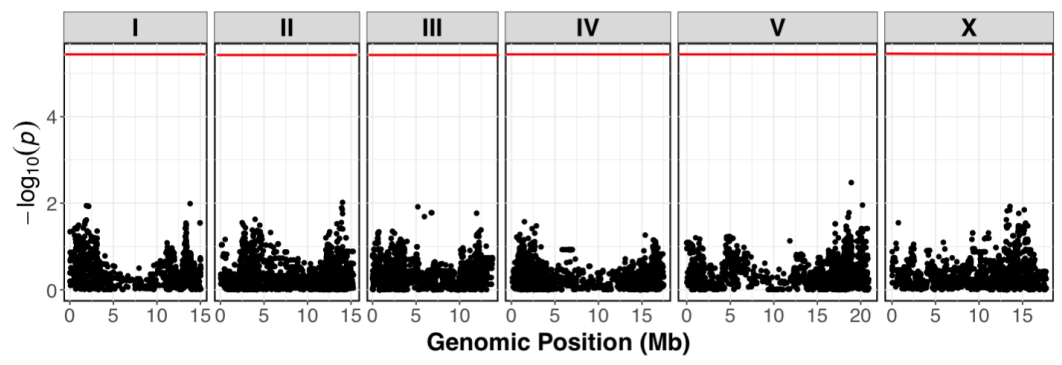

C Sperm size (CV, cross-sectional area)

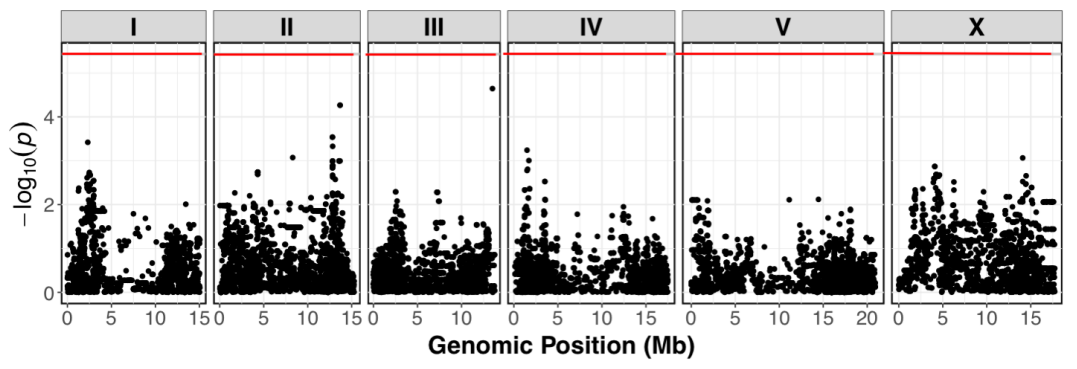

Figure 2. Genome-wide association mapping for $C$. elegans male sperm size

Manhattan plots of single-marker based GWA mappings show no significant genomic regions for least-squares mean estimates (LSM) of (A) sperm cross-sectional area, (B) sperm mean diameter and (C) CV (sperm crosssectional area). Each dot represents an SNV that is present in at least $5 \%$ of the assayed population. The genomic location of each SNV is plotted on the x-axis, and the statistical significance is plotted on the y-axis. The Bonferroni-corrected significance threshold is shown as a red horizontal line. 
Sperm size is a poor predictor of male competitive ability and reproductive performance Sperm size has been shown to be a central variable explaining differences in male reproductive success and competitive ability among C. elegans wild isolates (LaMunyon and Ward 1998; Murray et al. 2011; Wegewitz et al. 2008). We therefore tested whether the observed natural variation in $C$. elegans male sperm size correlates with variation in fertilization success and male competitive ability using eight strains with divergent male sperm size (from smallest to largest: LSJ1, JU561, CX11285, N2, CB4856, EG4946, JU782, JU393). Using similar assays described previously (Murray et al. 2011; Wegewitz et al. 2008), we measured the average number of inseminated fog- 2 females by a single male (Figure 3A) and resulting progeny counts (Figure 3C). For both measures, we found significant variation among strains (Figures $3 \mathrm{~A}$ and $3 \mathrm{C}$ ) but no correlation with sperm size (Figures 3B and 3D).

We further measured ejaculate size, as the number of sperm present in the uterus and spermatheca of fog-2 females after a single insemination event (Figure 3E). In contrast to a previous study on gonochoristic species Caenorhabditis species (Vielle et al. 2016), we did not find any evidence for a negative correlation between sperm size and ejaculate size (Figure $3 F$ ), which would be suggestive of a trade-off between sperm size and sperm number.

In addition, the polymorphic plugging phenotype of males, i.e. the deposition of a gelatinous plug on the vulva after copulation, likely representing a trait of male competitive ability as it affects mating ability of subsequent males (Palopoli et al. 2008; Hodgkin and Doniach 1997; Barker 1994), did not depend on sperm size as the average male sperm size did not differ between plugging versus non-plugging strains (ANOVA, $\mathrm{F}_{1,96}=0.09, \mathrm{P}=0.77$ ) (Table S3). 
A

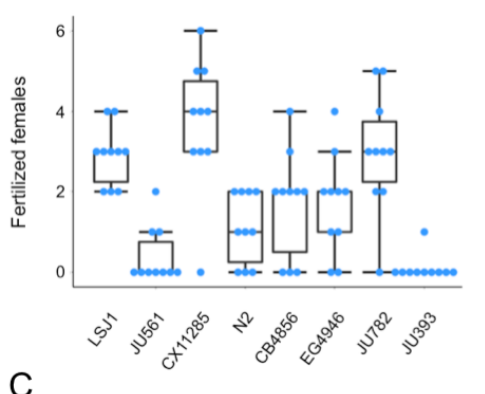

C

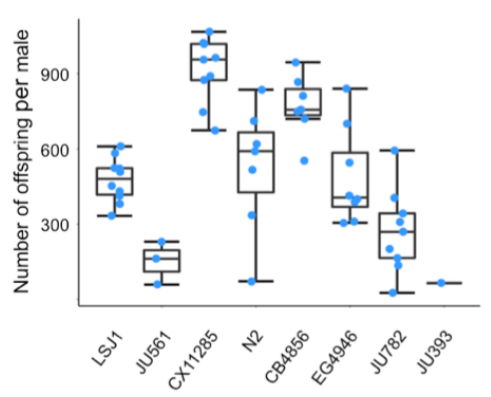

E

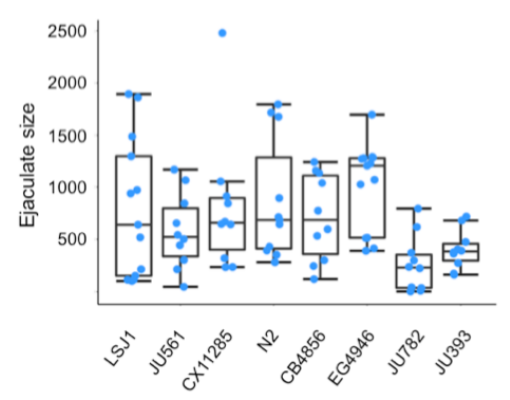

B
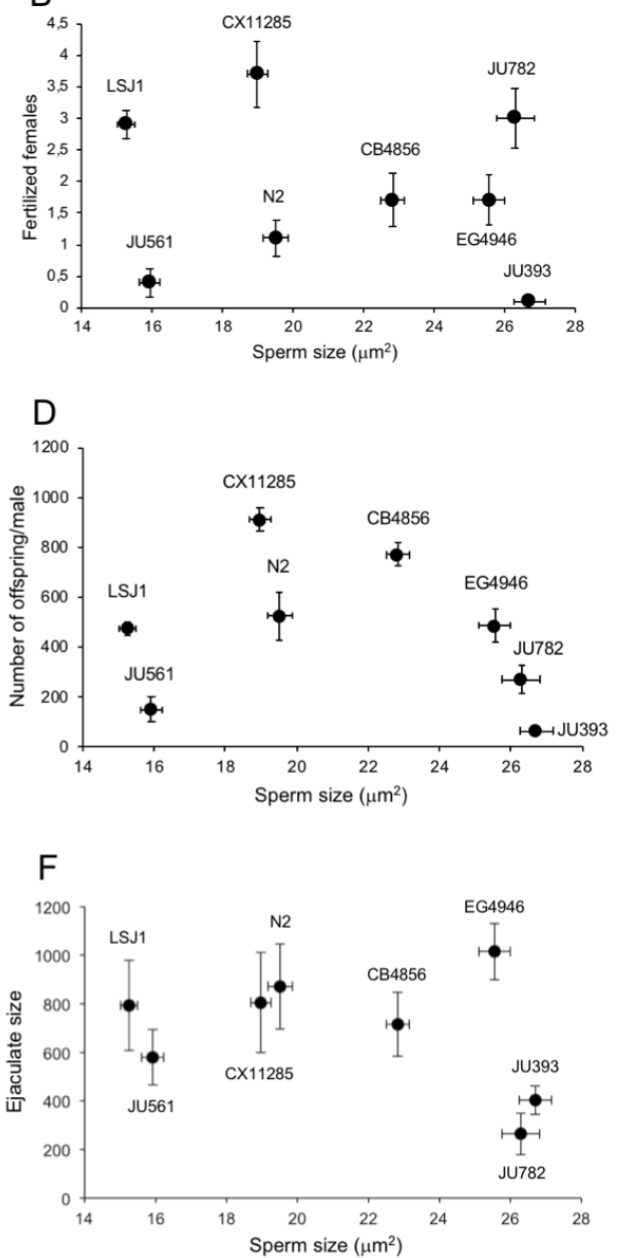
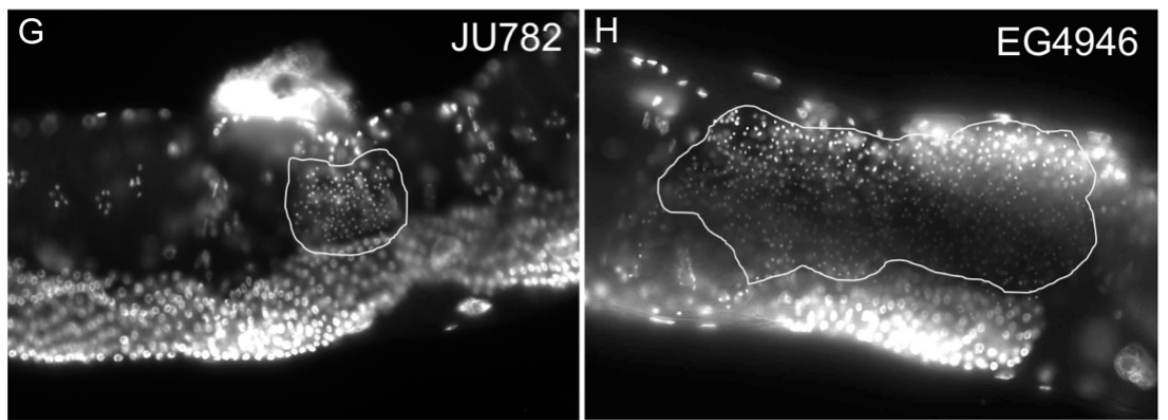

Figure 3. Differences in male mating ability and fertility between strains with variable male sperm size Male mating ability, fertility, and ejaculate size in eight C. elegans strains with different average male sperm size. Strains with smallest (LSJ1) to largest (JU393) male sperm are arranged from left to right. (A) Significant strain variation in the number of $f \circ g$ - 2 females fertilized by a single male during an eight hour window (KruskalWallis, $\left.\chi^{2}=45.12, \mathrm{df}=7, \mathrm{P}<0.0001\right)$ and $(\mathrm{B})$ absence of correlation with average male sperm size ( $\rho$ Spearman $=-$ $0.23, \mathrm{P}=0.59$ ). (C) Significant strain variation in the number of offspring sired by a single male during eight hours of mating with up to ten females (Kruskal-Wallis, $\chi^{2}=37.78, \mathrm{df}=7, \mathrm{P}<0.0001$ ) and (D) absence of correlation with average male sperm size ( $\rho$ Spearman $=-0.29, \mathrm{P}=0.49$ ). (E) Significant strain variation in ejaculate size, as measured by the number of sperm deposited by one male in a single mating (ANOVA, $\mathrm{F}_{7,86}=3.77$, $\mathrm{P}=0.0014)$ and $(\mathrm{F})$ no correlation between average ejaculate size and male sperm size ( $\rho$ Spearman $=-0.33, \mathrm{P}=0.42$, log-transformed data). (G, H) Images of DAPI-stained fog-2 females after single male mating event of strains (G) JU782 and (H) EG4946. Samples sizes were between 10 and 13 animals per strain per experiment. 
To complement the above experiments, we next asked whether variation in male sperm size may explain differences in male fertilization success when competing with (smaller) hermaphrodite self-sperm. In these experiments, young hermaphrodites of the strain CB4856 were allowed to mate once with a single male of the eight test strains. The proportion of resulting male progeny was then used as a measure of male fertilization success. Males of the eight strains showed significant differences in fertilization success when mated to CB4856 hermaphrodites (Figure 4A), however, there was no correlation between male fertilization success and sperm size across strains (Figure 4B). To test for differences in male-male competitive ability among the eight strains with different-sized sperm we used another, previously developed assay (Murray et al. 2011), in which fog-2 females were first mated once to males of the transgenic strain PD4790, expressing green fluorescent protein (GFP) in the pharynx. Subsequently, these inseminated females were mated a second time, to a single male of the eight test strains. Competitive ability (in fertilization success) of a given wild strain is thus indicated by the extent of GFP-negative offspring. Although we found extensive variation in competitive ability (Figure 4C), no correlation between this measure of male sperm competitive ability and sperm size was observed (Figure 4D). Time-course analysis of sperm precedence over four consecutive days after mating revealed additional differences among strains, however, without any clear connection to differences in sperm size. (Figure 4E). Taken together, these experimental results suggest that $C$. elegans male sperm size does not consistently correlate with male fertilization success or competitive ability (Murray et al. 2011), implying that other sperm, morphological, or behavioural traits need to be considered to account for natural variation in male competitive ability and overall male reproductive performance. Consistent with this idea, we find that sperm transfer during a single mating (ejaculate size) (Figure 3E) rather than sperm size shows a strong positive correlation with male fertility when mated to hermaphrodites (Figure 4F). 
A
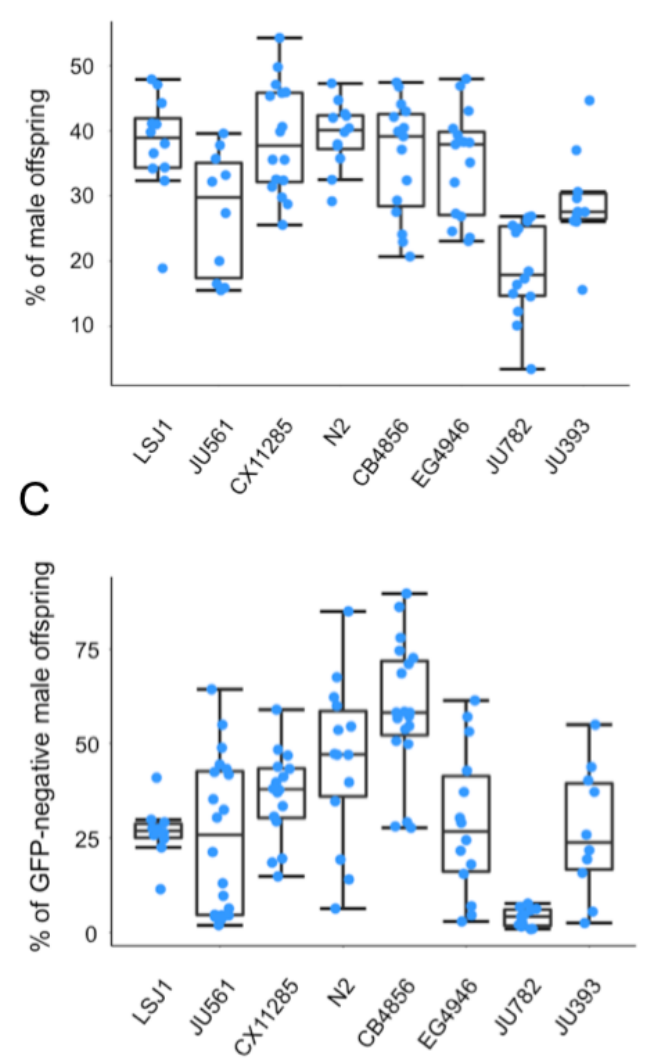

E

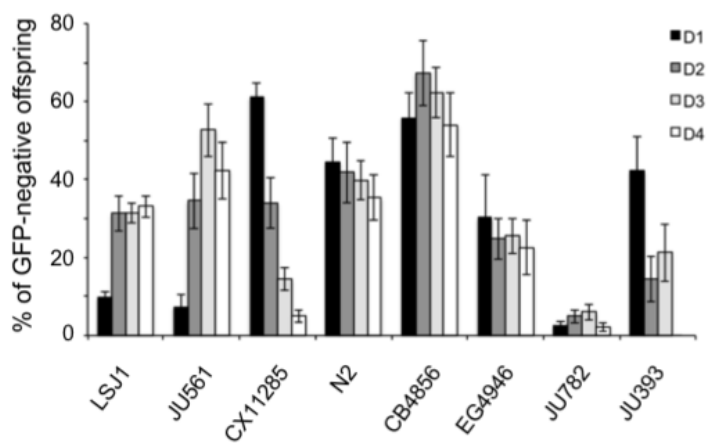

B

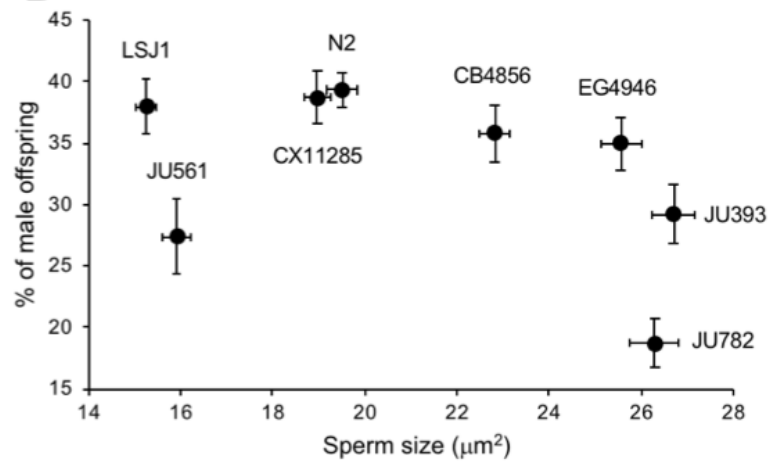

D
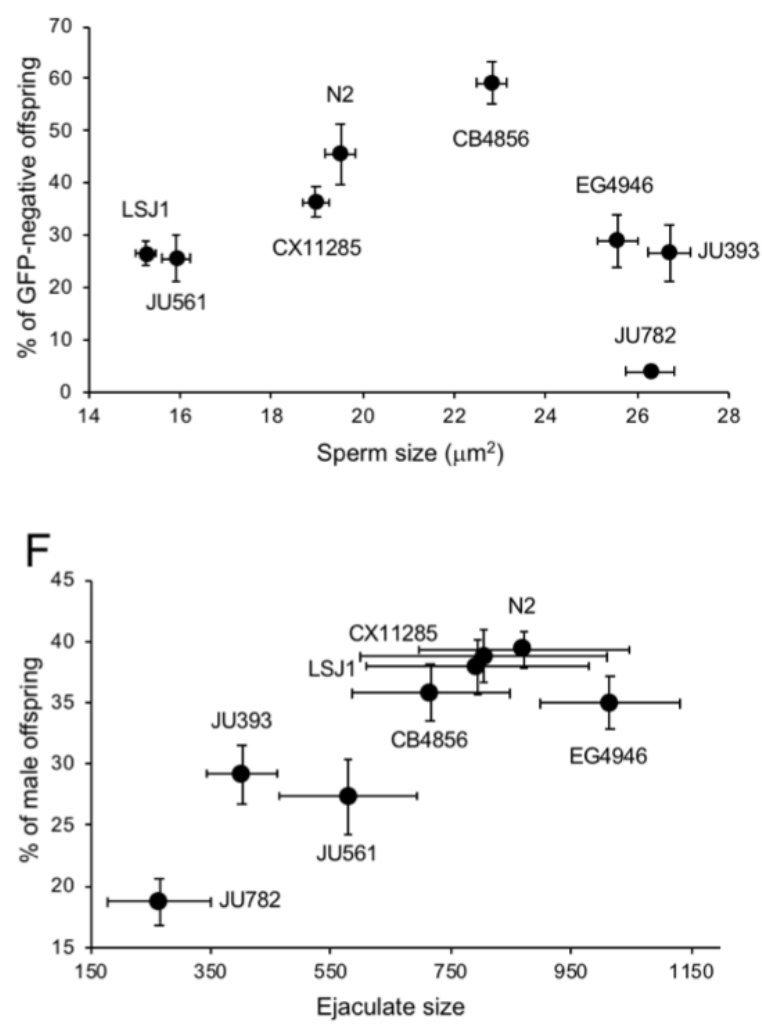

Figure 4. Differences in competitive ability between strains with variable male sperm size

Male competitive ability in fertilization success of eight $C$. elegans strains with different average male sperm size. Strains with smallest (LSJ1) to largest (JU393) sperm are arranged from left to right. (A) Significant strain variation in male fertilization success when competing with hermaphrodite self-sperm (strain CB4856) (ANOVA, $\mathrm{F}_{7,103}=11.20, \mathrm{P}<0.0001$ ). (B) No correlation between male fertilization success and male sperm size ( $\rho$ Spearman $=-0.45, P=0.45$ ). (C) Significant strain variation in male-male competitive ability (in fertilization success) sperm (ANOVA, $\mathrm{F}_{7,113}=14,66, \mathrm{P}<0.0001$ ). Male competitive ability of a given strain (versus the GFPpositive strain PD4790) quantified by the proportion of GFP-negative offspring produced over four days after mating. (D) No correlation between competitive ability and male sperm size ( $\rho$ Spearman $=0.00, \mathrm{P}=1)$. (E) Details of time course of progeny production across the four days after mating event (same data as in Figure 4C). (F) Significant correlation between ejaculate size (same data as in Figure 3E) and male fertilization success (Figure 4A) ( $\rho$ Spearman $=0.74, \mathrm{P}=0.036$ ). Samples sizes: $\mathrm{n}=10-20$ per strain per experiment. 


\section{Natural variation in hermaphrodite sperm size: no correlation with variation in male sperm size}

An unresolved question is whether genetic mechanisms in C. elegans sperm size determination are shared between the sexes, and more generally, to what extent homologous traits common to both sexes evolve. Hermaphrodite sperm is consistently smaller than male sperm in all three male-hermaphrodite species but also shows significant size variation across isolates within each species (Vielle et al. 2016). Previously, a weak positive correlation between the average sperm size of hermaphrodites and males was only found in C. tropicalis but not in C. elegans or C. briggsae (Vielle et al. 2016). This analysis was based on a small set of strains $(\mathrm{n}=5)$, so we revisited this question using 12 strains differing in male sperm size. In agreement with previous reports (Baldi et al. 2011; Vielle et al. 2016), hermaphrodite sperm showed significant genetic variation and was substantially smaller than male sperm in all strains (Figure 5A and Table S4). Again, as in Vielle et al. (2016), there was no significant cross-sexual correlation in sperm size (Figure 5B).

Given the presence of significant natural variation in C. elegans hermaphrodite sperm size, we tested whether this variation is linked to differential sperm production, indicative of a potential trade-off between hermaphrodite sperm size and sperm number. Across eight strains hermaphrodite sperm production differed significantly (ANOVA, $\mathrm{F}_{7,184}=5.37, \mathrm{P}<0.0001$ ) (Table S5), but we found no a negative correlation between hermaphrodite sperm size and number (Figure 5C).

Because sperm size and egg size show a weak positive correlation across (primarily gonochoristic) Caenorhabditis species (Vielle et al. 2016), we also tested for such a correlation across $C$. elegans strains. Using eggs size measurements from a previous study (Farhadifar et al. 2015), we did not detect any significant correlations, between either male sperm and egg size $\left(\mathrm{F}_{1,72}=0.26, \mathrm{P}=0.61\right)$ or between hermaphrodite sperm and egg size $\left(\mathrm{F}_{1,9}=0.62, \mathrm{P}=0.46\right)$. 

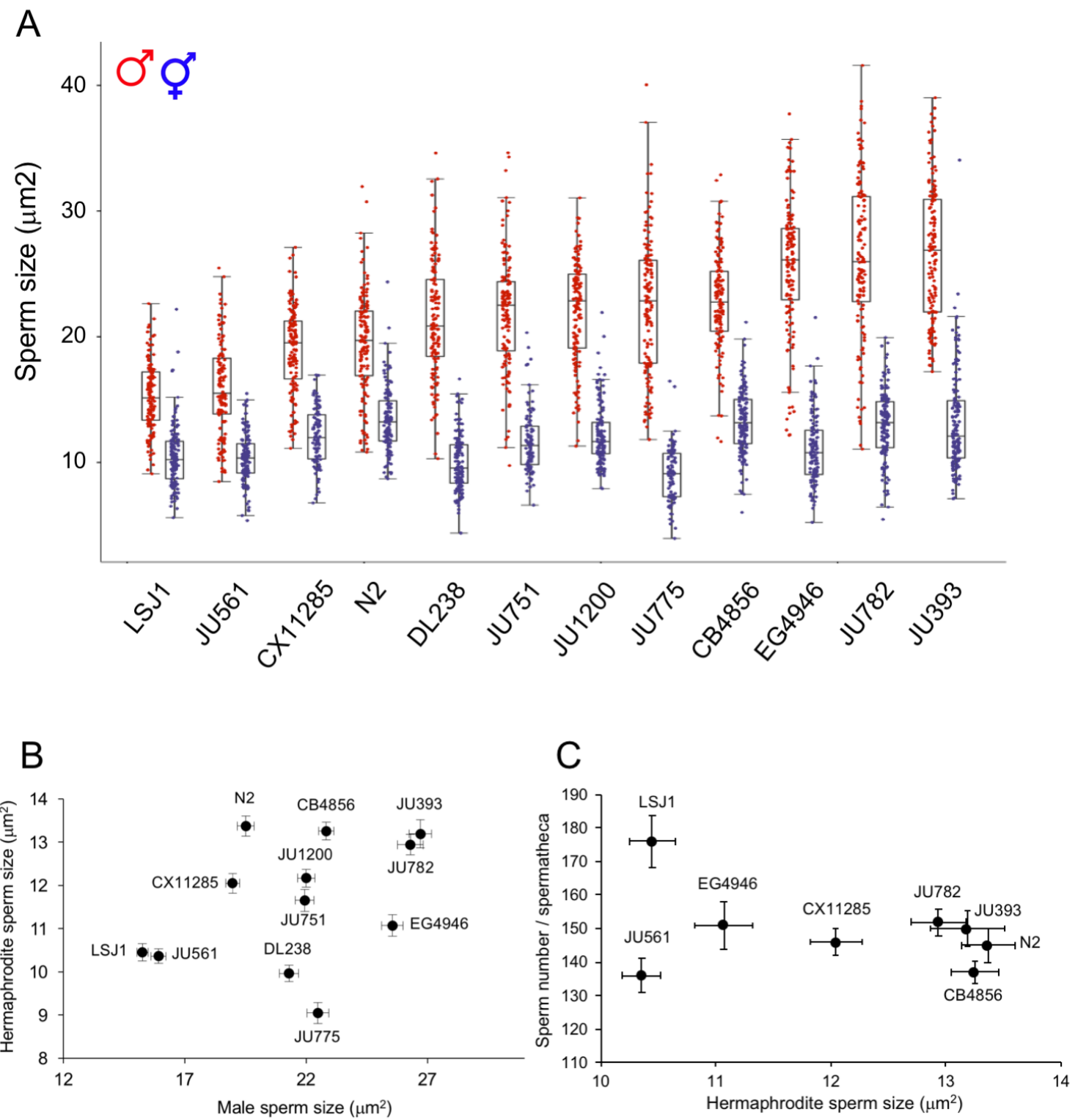

Figure 5. No correlation of $\boldsymbol{C}$. elegans male and hermaphrodite sperm size across strains

(A) Effect of strain genotype and sex on $C$. elegans sperm size (ANOVA, effect sex: $\mathrm{F}_{1,3207}=6267.55, \mathrm{P}<0.0001$; effect strain: $\mathrm{F}_{11,3207}=107.14, \mathrm{P}<0.0001$; interaction sex $\mathrm{x}$ strain: $\left.\mathrm{F}_{11,3207}=50.90, \mathrm{P}<0.0001\right)$. For each strain, 87152 hermaphrodite spermatids from 7-13 individuals were measured (male sperm size data from Figure 1). (B) The absence of a significant correlation between male and hermaphrodite sperm size was inferred from leastsquares regression of strain mean values $\left(\mathrm{F}_{1,12}=1.38, \mathrm{R}^{2}=0.11, \mathrm{P}=0.27\right)$. (C) No correlation between average hermaphrodite sperm size and sperm number across eight strains $(\rho$ Pearson $=-0.039, \mathrm{P}=0.40)$. Hermaphrodite selfsperm number were established by measuring all sperm contained within a single spermatheca of 12-31 individuals per strain.

\section{Allometric relationships between sperm and body size}

We next tested whether natural variation in C. elegans sperm size may reflect fixed allometric relationships between body and cell size. Such positive correlations between sperm size (length or cell size) and animal body size or mass have been frequently observed in diverse invertebrate taxa (Pitnick et al. 2009). Size of amoeboid sperm of nematodes, including Caenorhabditis, partially correlates with male body size across species (LaMunyon and Ward 1999; Vielle et al. 2016). In contrast, whether intraspecific variation in body size is linked to variation in sperm size in C. elegans, and other species, had so far not been evaluated. 
Measuring early adult body size of hermaphrodites and males in a subset of strains, we found significant variation across strains and sex (Table S6); however, we did not detect any positive correlation between average sperm size and body size (length) in either sex (Figures $6 \mathrm{~A}$ and $6 \mathrm{~B})$. In males only, sperm size was strongly correlated with body width $\left(\mathrm{F}_{1,10}=16.86\right.$, $\mathrm{R}^{2}=0.65, \mathrm{P}=0.0027$ ) (Figures $6 \mathrm{C}$ and $6 \mathrm{D}$ ). As male body width may simply increase due to storing of larger sperm and thus increased gonad width (Vielle et al. 2016), we found little evidence for a positive scaling relationship between male body size and sperm size.

A

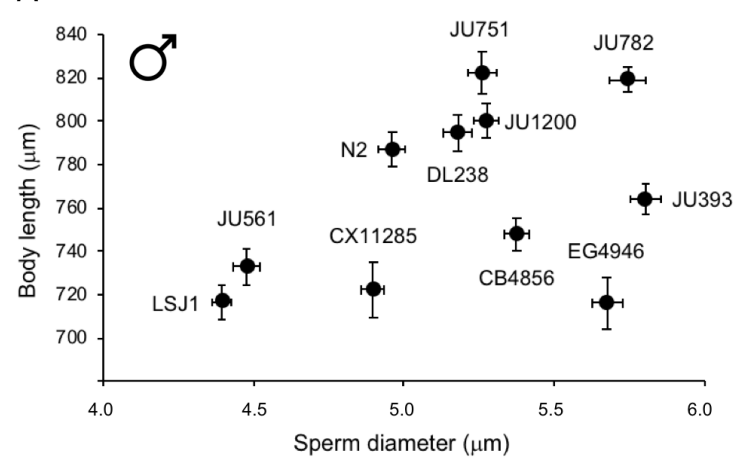

C

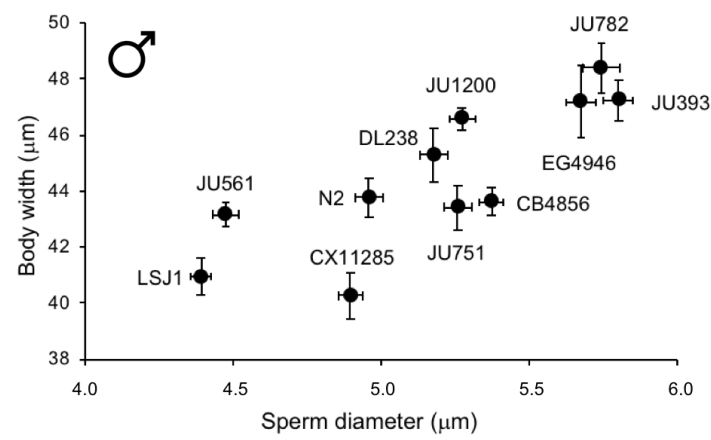

B

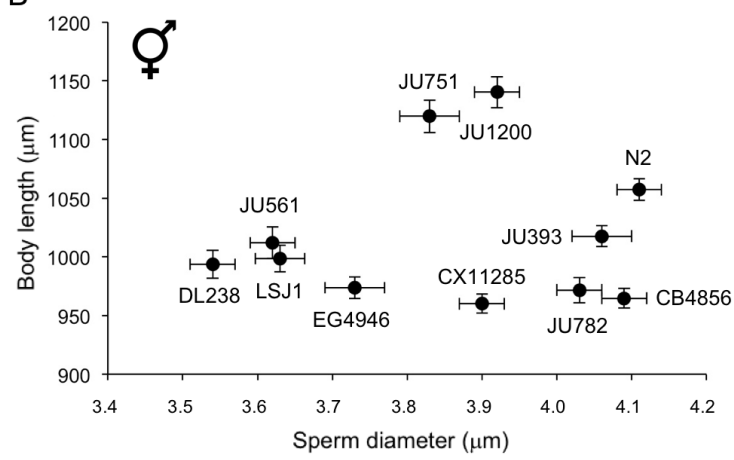

D

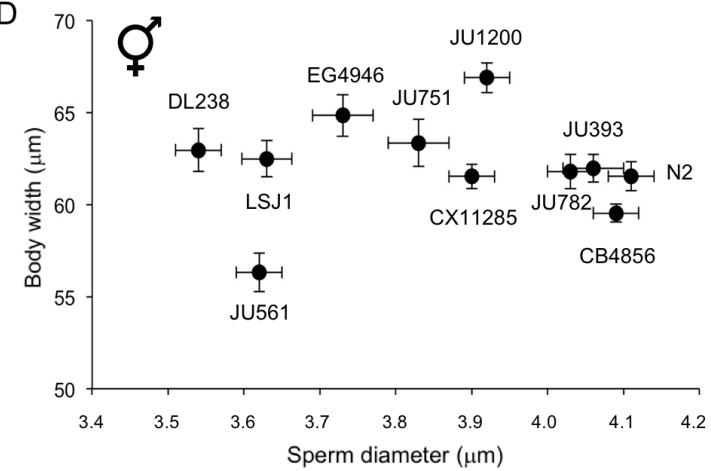

Figure 6. Allometric relationships between sperm and body size

Absence of scaling relationships between sperm size (mean diameter) and body length in $(A)$ males $\left(\mathrm{F}_{1,10}=1.88\right.$, $\left.\mathrm{R}^{2}=0.17, \mathrm{P}=0.20\right)$ and (B) hermaphrodites $\left(\mathrm{F}_{1,10}=0.005, \mathrm{R}^{2}=0.0005, \mathrm{P}=0.94\right)$. Significant positive association between body width and sperm size in $(C)$ males $\left(F_{1,10}=16.61, \mathrm{R}^{2}=0.65, \mathrm{P}=0.0028\right)$ but not (D) hermaphrodites $\left(F_{1,10}=0.43, R^{2}=0.05, P=0.53\right)$. Sperm size measures based on least-squares regression of strain mean (LSM); all data log-transformed for statistical analyses. Body length and width was measured in 10-29 individuals per strain and per sex (Table S6).

\section{Laboratory-derived strains LSJ1 and LSJ2 exhibit strongly reduced male and hermaphrodite sperm size}

Across all 97 C. elegans strains measured, LSJ1 exhibited the smallest male sperm size (Figure 1A). LSJ1 is a laboratory-derived strain and shares a common ancestor with the reference strain N2 (Figure 7A), which shows a much larger male sperm size (Figures 1A and 7B). The two lineages diverged between 1957-1958. N2 was then maintained on agar plates seeded with $E$. coli for approximately 15 years, while LSJ1 was maintained in axenic liquid culture for close to 40 years before cultures were cryopreserved. LSJ2 is a derivative of LSJ1 that was kept in liquid culture for another 14 years prior to freezing (McGrath et al. 2011; McGrath et al. 2009; Large et al. 2017; Sterken et al. 2015). We therefore also measured LSJ2, which displayed a similarly small sperm size as LSJ1 (Figure 7B). In contrast, the N2derived strain $\mathrm{CC} 1$, grown in liquid axenic medium for only four years, did not differ from N2 in male sperm size (Figure 7B). Given the common, inbred, and likely isogenic "Bristol 
ancestor" of LSJ and N2-CC1 lineages, these results suggest that the evolution of reduced male sperm size in the LSJ lineage occurred due to de novo mutations before 1995. Of note, LSJ1 and LSJ2 strain also showed significantly reduced hermaphrodite sperm size relative to N2 (and CC1) (Figure 7C).
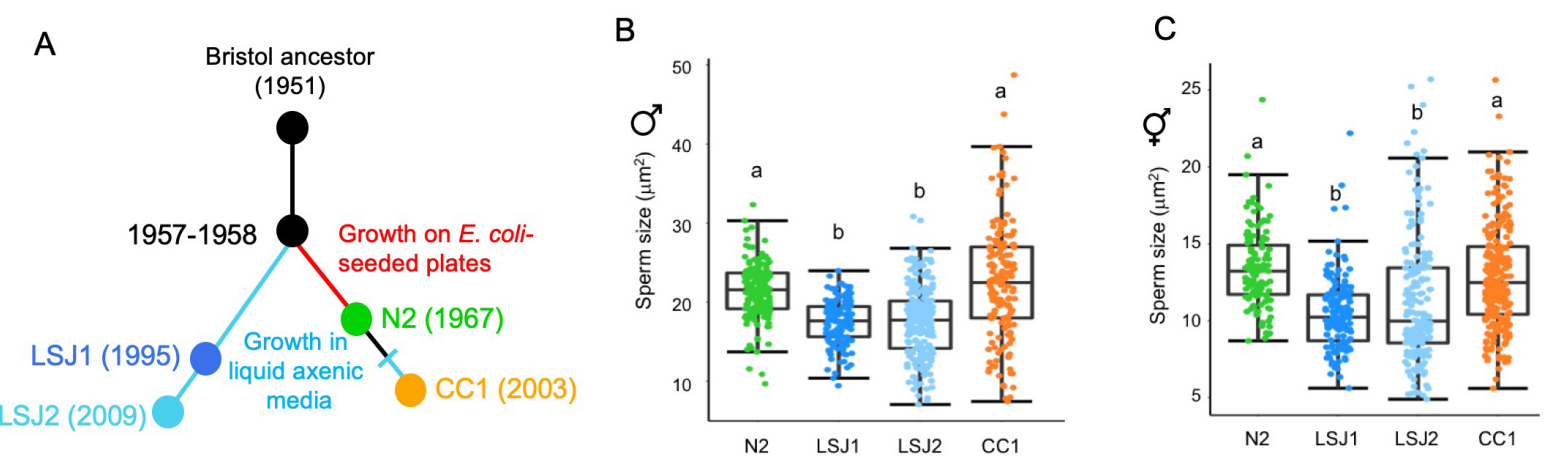

Figure 7. LSJ1 and LSJ2 strains exhibit strongly reduced male and hermaphrodite sperm size

(A) Laboratory evolution of LSJ and N2 lineages in the laboratory, after isolation of the common ancestral strain "Bristol", derived from a single hermaphrodite individual, in 1951. LSJ1 and LSJ2 were cultivated in axenic liquid medium and N2 was cultivated on agar plates (after McGrath et al. 2011). B) Male sperm size: LSJ1 and LSJ2 exhibit significantly reduced male sperm size compared to N2 and CC1 (ANOVA, effect strain: $\left.\mathrm{F}_{3,658}=65.62, \mathrm{P}<0.0001\right)$. (C) Hermaphrodite sperm size: LSJ1 and LSJ2 exhibit significantly reduced hermaphrodite sperm size compared to $\mathrm{N} 2$ and $\mathrm{CC} 1$ (ANOVA, effect strain: $\mathrm{F}_{3,666}=40.95, \mathrm{P}<0.0001$ ). For male sperm measurements, 135-225 sperm were analysed from 9-15 individuals of each strain. For hermaphrodite sperm measurements, 123-248 sperm were analysed from 9-19 individuals of each strain. Values labelled with different letters indicate significant differences (Tukey’s HSD, $P<0.05$ ).

\section{Reduced male sperm size of LSJ strains is caused by genetic variation in nurf-1}

Whole-genome short-read sequencing identified 188 and 94 new mutations fixed in the LSJ2 and N2 lineages, respectively (McGrath et al. 2011). These mutations include a 60 bp deletion in the 3' end of nurf-1, which encodes the orthologue of the BPTF subunit of the NURF chromatin remodelling complex (McGrath et al. 2011; Large et al. 2016; Andersen et al. 2006). This variant is predicted to replace the last 16 amino acids of the protein with 11 novel residues and is known to underlie multiple life history differences between N2 and LSJ2, including reproductive timing, progeny production, growth rate, lifespan and dauer formation (Large et al. 2017; Large et al. 2016). Moreover, the NURF complex has previously been shown to function in C. briggsae spermatogenesis (Chen et al. 2014) as well as Drosophila spermatogenesis (Kwon et al. 2009). We thus reasoned that the nurf-1 deletion specific to the LSJ lineage provides a good candidate explaining reduced male (and hermaphrodite) sperm size. This hypothesis was supported by the observation that RNAi knock-down of nurf-1 resulted in a significant reduction of male sperm size in the N2 strain (Figure S1). To further test our hypothesis, we first examined the introgression line CX13248 (kyIR87) containing the LSJ2 region surrounding nurf-1 in an N2-like background (CX12311, which is of N2 genotype except for introgressed $n p r-1$ and $g l b-5$ alleles from the strain CB4856) (McGrath et al. 2011). The kyIR87 introgression contains the 60 bp deletion along with LSJ2 alleles of eight additional variants, including an SNV in the intron of nurf-1 that was fixed in the N2 lineage (Large et al. 2016). Consistent with our hypothesis, sperm size of the CX13248 strain containing the nurf-1 deletion was significantly smaller compared to CX12311, both in males (Figure 8A) and hermaphrodites (Figure 8B). In addition, sperm size of the nurf-1(n4295) deletion mutant (N2 background) (Andersen et al. 2006) was also strongly reduced in both 
sexes (Figures 8C and 8D); therefore, strains containing two different deletions in the 3' coding region of nurf-1 result in reduced C. elegans sperm size. Specifically, the 60 bp nurf-1 deletion of LSJ strains covers the 3' coding region (plus stop codon and $8 \mathrm{bp}$ of the 3' UTR region), and the nurf-1(n4295) deletion spans 1078 bp of the 3' coding region (Large et al. 2016). Importantly, both deletions impact the same 13 (out of a total of 16) nurf-1 isoforms.

To test whether nurf-1 is the causal gene underlying reduced sperm size in the LSJ lineage, we performed a quantitative complementation test (QCT) (Long et al. 1996) taking advantage of the two nurf-1 deletion alleles present in LSJ1 and nurf-1(n4295) (Figure 8E). The recessive phenotype caused by either nurf-1 deletion was confirmed by the large, N2-like sperm size in F1 males derived from crosses between N2 and LSJ1 and between N2 and nurf1(n4295) (Figure 8E). F1 males derived from bi-directional crosses between LSJ1 and nurf1(n4295), however, exhibited small sperm size, comparable to parental strains (Figure 4B). We conclude that variation in the gene nurf- 1 underlies the evolution of reduced male sperm size in LSJ strains.

Collectively, our experiments suggest that the 60 bp deletion in nurf- 1 is the causal variant responsible for the decreased sperm size in males and hermaphrodites in the LSJ lineage. First, quantitative complementation indicates nurf-1 to be the causal gene. Second, the LSJ1/LSJ2 strains are outliers with regards to sperm size, suggesting that a mutation occurred in this lineage, like the $60 \mathrm{bp}$ deletion, to reduce sperm size. Finally, the $n 4295$ allele, which phenocopies the LSJ strains, is genetically similar to the $60 \mathrm{bp}$ deletion, affecting the C-terminus of the protein. However, the intron SNV in nurf-1, derived in the N2 lineage, cannot be completely ruled out to explain observed sperm size differences.
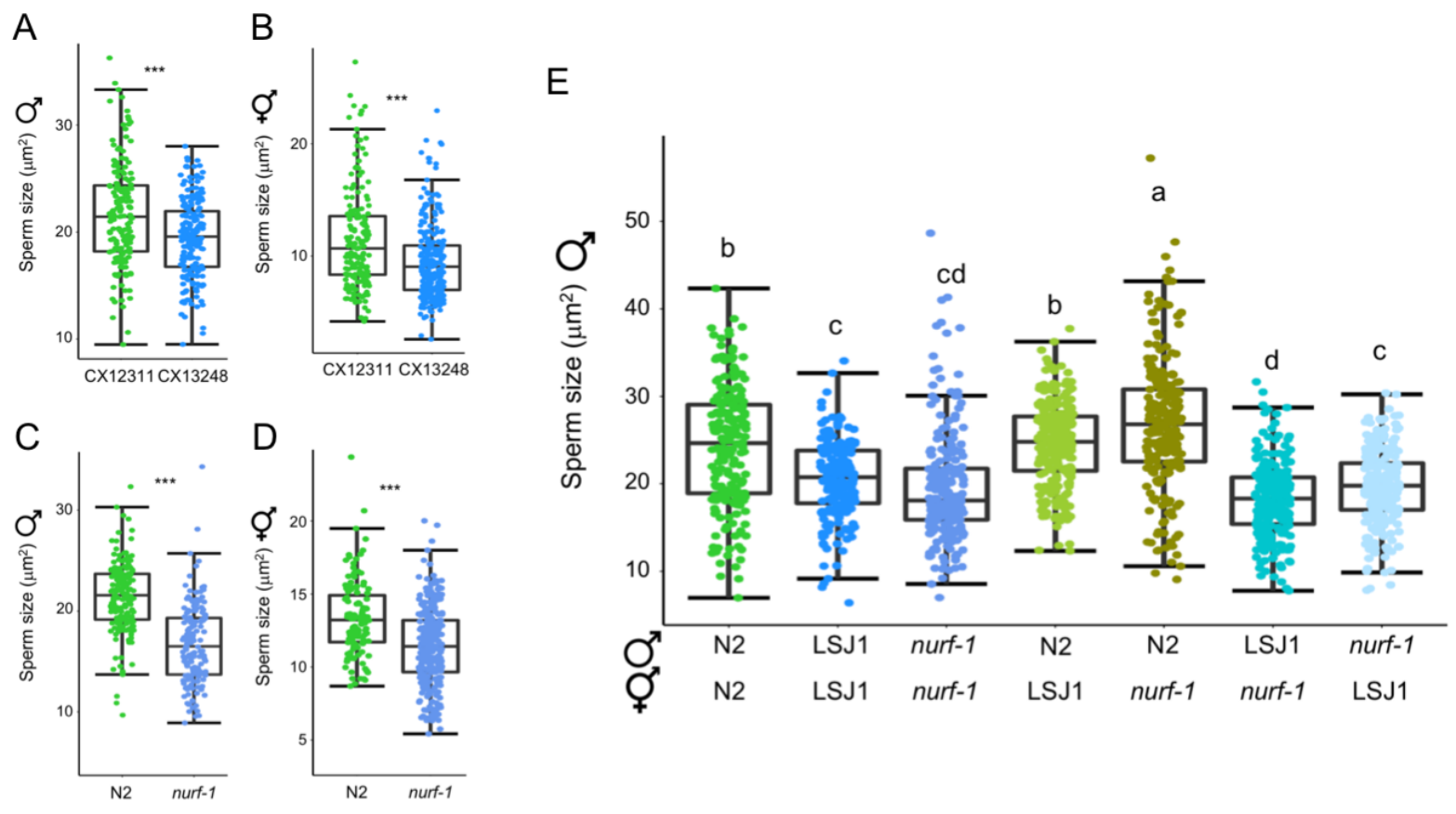

Figure 8. Reduced male sperm size of LSJ strains is caused by variation in nurf-1

(A, B) A near-isogenic line (CX13248) with the LS2 genomic region containing the nurf-1 deletion exhibits reduced sperm size relative to the N2-like parent $C X 12311$ in $(A)$ males (ANOVA, $F_{1,336}=23.11, P<0.0001$ ) and (B) hermaphrodites (ANOVA, $\mathrm{F}_{1,420}=30.35, \mathrm{P}<0.0001$ ). (C, D) Sperm size of the deletion mutant nurf-1(n4295) is reduced in (C) males (ANOVA, $\mathrm{F}_{1,322}=184.30, \mathrm{P}<0.0001$ ) and (D) hermaphrodites (ANOVA, $\mathrm{F}_{1,383}=61.64$, $\mathrm{P}<0.0001$ ). (E) Quantitative complementation tests using the strains N2, LSJ1, and nurf-1(n4295) (ANOVA, effect strain: $\left.\mathrm{F}_{6,1502}=97.09, \mathrm{P}<0.0001\right)$. Values labelled with different letters indicate significant differences (Tukey's HSD, $P<0.05$ ). 


\section{Mutants of different NURF complex components exhibit reduced sperm and spermatocyte size}

Observed sperm size reduction caused by the two independent nurf-1 deletions implies a potential role of the NURF chromatin remodelling complex in C. elegans sperm size determination. We therefore tested whether mutants of other complex members, including PYP-1, RBA-1 and the ATPase component ISW-1 (Figure 9A) (Tsukiyama et al. 1995; Andersen et al. 2006) show altered sperm size. Previously isolated deletion mutants of pyp-1 and $i s w-1$ (Andersen et al. 2006) exhibited strongly reduced male sperm size, with isw-1 mutants showing the most extreme sperm size reduction of all tested NURF complex mutants (Figure 9B). Using the $i s w-1$ (n3297) allele (Andersen et al. 2006), we further confirm that hermaphrodite sperm size was significantly reduced compared to the N2 wild-type (Figure S2).

Overall germline structure and organisation of strains with small sperm size (including LSJ1 and LSJ2) appeared intact, except for a fraction of isw-1 mutant individuals that displayed severe errors, such as displacement of spermatids into the distal region. Given that Caenorhabditis sperm size differences are developmentally established at the primary spermatocyte stage (Vielle et al. 2016), we measured primary male spermatocyte size in isw1(n3297) animals with intact germline structure: primary spermatocyte size was on average significantly smaller than in the wild-type N2 strain (Figures 9C to 9E). Size variation of $C$. elegans sperm size observed in NURF complex mutants was thus introduced prior to, or at, the primary spermatocyte stage, as observed for sperm size variation occurring within and between different Caenorhabditis species (Vielle et al. 2016). Taken together, these data suggest that the NURF chromatin remodelling complex likely acts, directly or indirectly, in $C$. elegans sperm size determination. Furthermore, the role of nurf-1 in sperm size determination seems to be evolutionarily conserved because nurf-1 RNAi also reduced sperm size of a gonochoristic Caenorhabditis species, C. plicata, exhibiting substantially larger sperm (Vielle et al. 2016) (Figure S3). 
A
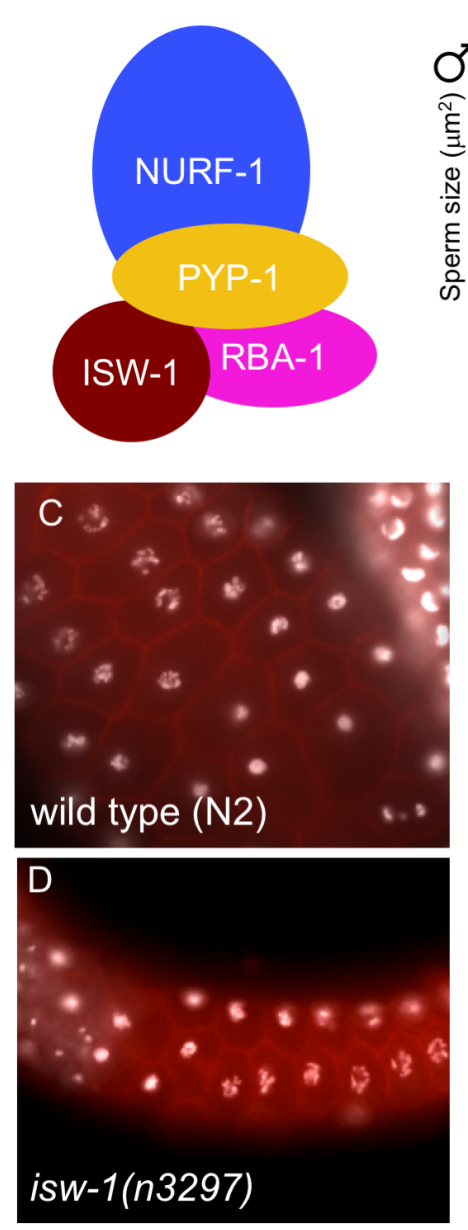

B
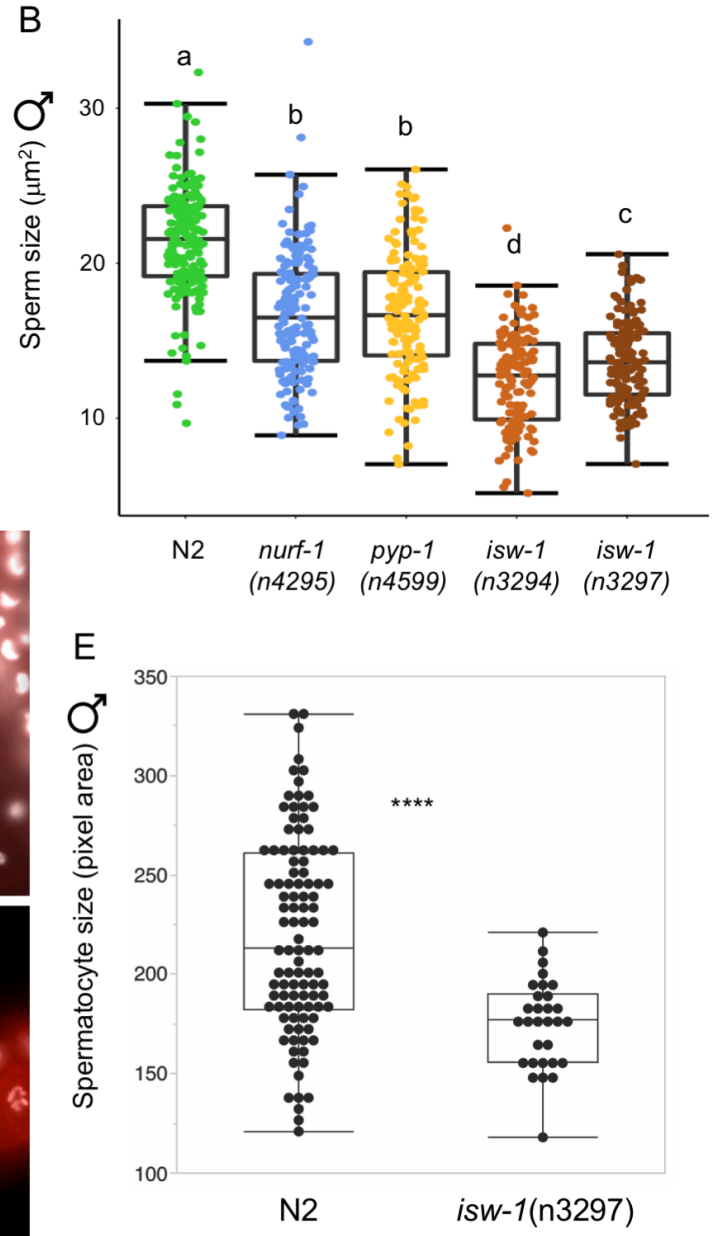

Figure 9. Mutants of the NURF complex exhibit reduced male sperm size and primary spermatocytes (A) Illustration of NURF complex components. (B) Mutation of different genes in the NURF complex reduce male sperm size (ANOVA, effect strain: $\mathrm{F}_{4,728}=192.23, \mathrm{P}<0.0001$ ). (C,D) Microscopy images of primary spermatocytes in (C) wild type (N2) and (D) isw-1(n3297) (DAPI: white, Phalloidin: red). D) Primary spermatocytes (area measurements) of the mutant isw-1(n3297) are significantly smaller than in the wild type (N2) (ANOVA, effect strain: $\mathrm{F}_{1,119}=83.47, \mathrm{P}<0.0001$ ).

\section{Male sperm size is reduced independently of body size in NURF-complex mutants}

An earlier study has shown that the 60 bp deletion in nurf-1 is responsible for reduced hermaphrodite body length in LSJ2, and similarly, the deletion allele nurf-1(n4295) was shown to exhibit a significantly reduced hermaphrodite body length relative to N2 (Large et al. 2016). We therefore hypothesized that perturbing activity of the NURF chromatin complex may cause systemic size reduction of diverse tissues and organs, including spermatids. Inconsistent with this hypothesis, we found male body size of nurf-1(n4295) with small sperm size to be significantly larger, rather than smaller than in the wild-type N2 strain (Figure 10); in addition, the $i s w-1$ (n3294) mutant with very small sperm had the same male body size as N2 (Figure 10). Male sperm size reduction in NURF mutants thus occurs independently of body size, suggesting that reduced male sperm size of LSJ strains is not necessarily a pleiotropic consequence of body size reduction in male body size. 
A

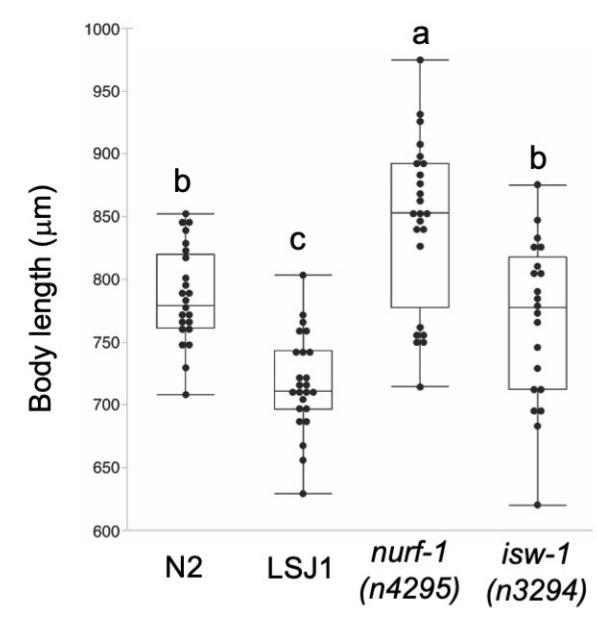

B

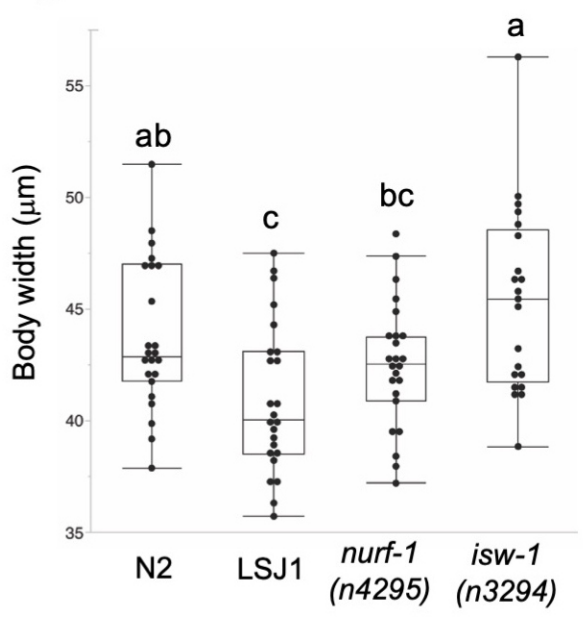

Figure 10. Male sperm size reduction in NURF mutants occurs independently of body size Significant differences in body size of strains N2, LSJ1, nurf-1(n4295) and isw-1(n3294). (A) Body length (ANOVA, $\mathrm{F}_{3,91}=23.36, \mathrm{P}<0.0001$ ). (B) Body width (ANOVA, $\mathrm{F}_{3,91}=6.83, \mathrm{P}=0.0003$ ). Values with different letters indicate significant differences (Tukey's HSD, $P<0.05$ ).

\section{DISCUSSION}

Our survey of intraspecific variation in C. elegans male sperm size uncovered significant heritable variation for this trait associated with sperm competitive ability. However, examining a subset of strains with divergent male sperm size, we did not detect a strong effect of male sperm size on competitive ability and reproductive performance. The second part of our study focused on the genetic basis underlying the evolutionary reduction in sperm size of laboratory-adapted strains, LSJ1 and LSJ2, which exhibit the smallest sperm size among all strains examined. This analysis, by means of mutants and quantitative complementation tests, shows that genetic changes in the nucleosome remodelling factor nurf-1 underlie the evolution of small male sperm size in the LSJ lineage. These and additional experimental results suggest that the NURF chromatin remodelling complex acts in C. elegans sperm size regulation.

\section{Natural variation in C. elegans male sperm size}

Although we observed significant heritable variation in C. elegans male sperm size, the vast majority of isolates show a relatively narrow average sperm size range, from $20 \mu \mathrm{m}^{2}$ and 25 $\mu \mathrm{m}^{2}$, and the only two significant outliers were the strains JU561 and LSJ1, with reduced male sperm size $\left(\sim 15 \mu \mathrm{m}^{2}\right)$ (Figure 1). Performing a genome-wide association study to detect potential genetic loci explaining variation in male sperm size did not yield any QTL, likely due to low statistical power and/or complex genetic trait architecture. As previously observed for some C. elegans isolates and other Caenorhabditis species (Vielle et al. 2016), male sperm size showed extreme variability within single individuals, and to a lesser extent, among populations of genetically identical individuals (Figure 1). Such within-genotype variability is often observed for sperm size traits, such as sperm length in taxa with flagellate sperm (Ward 1998; Morrow and Gage 2001b; Miller et al. 2003; Joly et al. 2004). Possibly, such high sperm size variance could reflect a means to maximize both average size and number of sperm produced, or developmentally decreasing sperm size variance may come at the cost of reduced sperm production speed (Parker and Begon 1993; Gomendio et al. 2006; Vielle et al. 2016). 


\section{Sperm size poorly predicts male competitive ability and reproductive performance}

Given the observation of heritable variation in C. elegans male sperm size - and the expectation that larger male sperm is more competitive - we asked whether this variation translated into differential competitive ability and reproductive success. In contrast to a previous study (Murray et al. 2011), we did not detect any significant correlations between male sperm size and male fertility or competitive ability (Figures 3 and 4). This unexpected outcome may be simply due to chance, given the small number of strains examined $(n=8)$; nevertheless, this result shows that male sperm size is unlikely to be the only key determinant of male reproductive success, so that other traits than sperm size may be important for male fertilization success or competitive ability. Consistent with this interpretation, the number of sperm transferred per mating (ejaculate size), which also differed significantly among strains, was strongly correlated with the number of offspring sired (Figure 4F). Although we did not detect a potential trade-off between male sperm size and ejaculate size, an earlier study (Murray et al. 2011) did report that the rate of sperm production was reduced in C. elegans strains with larger male sperm. Trade-offs between male sperm size and sperm number are thus likely to shape and limit the extent of sperm size evolution. Together with earlier studies (Hansen et al. 2015; Vielle et al. 2016), our study suggests that size is not necessarily the central sperm trait determining male competitive ability and fertilization success. Moreover, many other traits that we did not measure here, including male mating behaviour or hermaphrodite receptivity, will contribute to overall male competitive ability and reproductive performance - as such traits may also vary across $C$. elegans strains, they could mask the relative importance of sperm size variation in determining male reproductive success.

In addition, the observed absence of a tight relationship between male sperm size and competitive ability might also represent a signature of reduced male conflict and sperm competition, in line with reports of low rates of outcrossing in C. elegans wild populations (Barrière and Félix 2005, 2007; Félix and Braendle 2010) and reduced maintenance of (male) mating function (Chasnov 2013; Chasnov and Chow 2002; Thomas et al. 2012; Yin et al. 2018; Teotonio et al. 2006). Nevertheless, C. elegans male sperm seems to maintain the evolutionary potential to respond to changes in the extent of male mating competition: several experimental evolution studies have shown that male sperm size may rapidly evolve towards increased size in response to increased male-male competition (LaMunyon and Ward 2002; Palopoli et al. 2015; Poullet et al. 2016).

\section{C. elegans sexual sperm size dimorphism}

We also aimed to assess to what extent $C$. elegans sperm size differs between sexes, given that little is known about natural genetic variation in hermaphrodite sperm size and whether male and hermaphrodite sperm size may correlate. Specifically, a significant correlation between male and hermaphrodite sperm size could be indicative of shared genetic regulation of sperm size across sexes. Our analyses of intraspecific variation in C. elegans hermaphrodite sperm size confirmed previous reports (LaMunyon and Ward 1995, 1998; Ward and Carrel 1979; Vielle et al. 2016) that for any given strain, hermaphrodite sperm is always significantly smaller than male sperm (Figure 5A). As for male sperm size, we detected substantial levels of heritable variation in hermaphrodite sperm size. Although the two strains with smallest male sperm (LSJ1 and JU561) also had the smallest hermaphrodite sperm, male and hermaphrodite sperm size did not correlate across examined strains sperm (Figure 5). The C. elegans sperm size dimorphism is thought to reflect differential selection on conflicting sex-specific size optima: larger male sperm to increase sperm competitive ability versus smaller hermaphrodite sperm to accelerate sperm production to allow for a rapid switch to oogenesis, which is critical for early maturity and reproduction (Hodgkin and 
Barnes 1991; Cutter 2004); C. elegans hermaphrodites are protandrous with initial production of sperm, stored in the spermathecae, before switching to oocyte production. Therefore, the sequential spermatogenesis-oogenesis switch in $C$. elegans causes a trade-off between maximal sperm number (i.e. potential number of self-progeny) and minimal age at maturity (i.e. generation time). Consequently, evolution of small hermaphrodite sperm size results from selection for rapid self-sperm production, consistent with the fact that hermaphrodite sperm is always drastically smaller than male sperm (Figure 5A). In the same fashion, evolution of increased hermaphrodite sperm production may lead to smaller self-sperm. However, we did not find evidence for a trade-off between hermaphrodite sperm size across a set of $C$. elegans strains differing in sperm size (Figure 5C). Importantly, the evolution of small hermaphrodite sperm in androdioecious species seems not only to result from selection for improved self-fertilization but also from direct developmental effects, when spermatogenesis takes place in a female soma, that reduce hermaphrodite sperm size (Baldi et al. 2011). Disentangling these different evolutionary and developmental mechanisms in shaping C. elegans sperm size dimorphism therefore remains a major challenge.

\section{Genetic variation in nurf-1 explains the evolution of small sperm during laboratory adaptation}

Surprisingly, we uncovered extreme divergence in male sperm size between two very closely related C. elegans laboratory lineages, N2 versus LSJ1/LSJ2. Given their recent evolutionary divergence, and thus close genetic similarity (McGrath et al. 2011), we successfully applied a candidate approach to demonstrate that variation in the gene nurf-1, encoding a subunit of the NURF chromatin remodelling complex, explains reduced LSJ sperm size. The evolution of nurf- 1 function in the LSJ lineage underlies laboratory adaptation to the axenic liquid medium (Large et al. 2017). Specifically, the key causal molecular variant underlying improved LSJ hermaphrodite reproduction in this environment (relative to N2) is the $60 \mathrm{bp}$ nurf-1 deletion (Large et al. 2016), which we found to be also responsible for reduced sperm size. This deletion has been shown to have highly pleiotropic effects on life history traits, including reproduction, growth rate, life span and dauer formation (Large et al. 2016). Our result suggests that these life history changes may be, at least partly, mediated by changes in nurf-1 function in the germline, affecting sperm size, likely also in hermaphrodites. Furthermore, evolution of reduced hermaphrodite sperm size could thus be directly linked to the observed evolutionary increase in hermaphrodite sperm number (Figure 5C), although this remains to be experimentally verified; we did not formally show that the reduced hermaphrodite sperm size is due to the same genetic variant(s) in nurf-1 as in males. Given the previous demonstration that nurf-1 variants specific to the LSJ lineage confer improved fitness of hermaphrodites, the evolution of reduced male sperm size in the LSJ lineage likely reflects a pleiotropic consequence stemming from selection on hermaphrodite function. Moreover, because C. elegans males are generally incapable of mating in liquid culture, it appears likely that laboratory evolution of the LSJ lineage likely occurred in the complete absence of outcrossing. This result illustrates how selection for improved C. elegans hermaphrodite function (Large et al. 2016) can cause the deterioration of male-specific fitness trait (sperm size) through a specific sexually antagonistic genetic variant. Evolutionary variation at the nurf-1 gene can thus be considered a potential example of intralocus sexual conflict (Bonduriansky and Chenoweth 2009). Consistent with this interpretation, we observed that LSJ1 showed significantly reduced male-male competitive ability relative to N2 (Figures 4C and $4 \mathrm{E}$ ). On the other hand, despite the presumed absence of outcrossing over many hundreds of generations in liquid culture, we also found that LSJ1 male sperm function and male mating ability remained largely preserved (Figures 3 and 4). These observations exemplify how essential male functions in $C$. elegans can be maintained despite very rare outcrossing, 
i.e. strongly relaxed selection, due to pleiotropic effects, in particular, selection on hermaphrodite sperm function as sperm genes are shared by both sexes.

\section{A role for the NURF chromatin remodelling complex in $C$. elegans sperm size determination}

The observation that evolutionary reduction of $C$. elegans male sperm size is caused by variation in nurf- 1 suggests that this gene acts in $C$. elegans sperm size determination. Our analysis of multiple mutants in NURF-complex genes (nurf-1, isw-1, pyp-1), all of which displayed strongly reduced sperm size, not only in males but also in hermaphrodites, confirms this idea. As a subunit of the NURF chromatin remodelling complex, NURF-1 is a BPTF orthologue, involved in histone modification on nucleosomes and remodelling of nucleosomes through recruitment of ISWI, to modulate transcription (Badenhorst et al. 2002; Andersen et al. 2006; Ruthenburg et al. 2011; Wysocka et al. 2006). Members of the $C$. elegans NURF chromatin remodelling complex are expressed in diverse tissues and organs, including the developing germline (Craig et al. 2013; Feng et al. 2012; Reece-Hoyes et al. 2007). Consistent with these expression patterns, nurf-1 mutations affect diverse phenotypes in C. elegans, ranging from vulval development (Andersen et al. 2006) to multiple life history traits (Large et al. 2016). Therefore, whether the NURF chromatin remodelling complex specifically acts in the $C$. elegans germline to regulate expression of target genes modulating sperm size remains to be tested. Nevertheless, given the combined experimental evidence that NURF-1 activity affects (a) sperm size (this study), (b) C. briggsae spermatogenesis (Chen et al. 2014), and (c) reproductive timing and number of self-progeny (Large et al. 2016) clearly points to a specific role of the NURF chromatin remodelling in C. elegans sperm phenotypes.

\section{ACKNOWLEDGEMENTS}

We thank Asher Cutter, Marie-Anne Félix and Henrique Teotonio for discussion and comments on previous versions of the manuscript. Strains and materials were provided by the groups of Julie Ahringer, Cori Bargmann, Marie-Anne Félix, the C. elegans Natural Diversity Resource (CeNDR) (Cook et al. 2017), and the Caenorhabditis Genetics Center (CGC), which is funded by NIH Office of Research Infrastructure Programs (P40 OD010440). CB, $\mathrm{CG}$ and $\mathrm{AV}$ acknowledge financial support by the Centre National de la Recherche Scientifique (CNRS). NS was supported by an Erasmus mobility fellowship provided by the European Commission. SZ was supported by the National Institutes of Health Cell and Molecular Basis of Disease training grant (T32GM008061) and a Bernard and Martha Rappaport Fellowship. PTM was supported by NIH grant R01GM114170. ECA was supported by an American Cancer Society Research Scholar Grant (127313-RSG-15-135-01DD).

\section{REFERENCES}

Alcorn, M.R., D.C. Callander, A. Lopez-Santos, Y.N. Torres Cleuren, B. Birsoy et al., 2016 Heterotaxy in Caenorhabditis: widespread natural variation in left-right arrangement of the major organs. Philos Trans R Soc Lond B Biol Sci 371 (1710).

Andersen, E.C., J.P. Gerke, J.A. Shapiro, J.R. Crissman, R. Ghosh et al., 2012 Chromosomescale selective sweeps shape Caenorhabditis elegans genomic diversity. Nat Genet 44 (3):285-290.

Andersen, E.C., X. Lu, and H.R. Horvitz, 2006 C. elegans ISWI and NURF301 antagonize an $\mathrm{Rb}$-like pathway in the determination of multiple cell fates. Development 133 (14):2695-2704. 
Anderson, J.L., L.T. Morran, and P.C. Phillips, 2010 Outcrossing and the maintenance of males within C. elegans populations. J Hered 101 Suppl 1:S62-74.

Ashe, A., T. Belicard, J. Le Pen, P. Sarkies, L. Frezal et al., 2013 A deletion polymorphism in the Caenorhabditis elegans RIG-I homolog disables viral RNA dicing and antiviral immunity. Elife 2:e00994.

Badenhorst, P., M. Voas, I. Rebay, and C. Wu, 2002 Biological functions of the ISWI chromatin remodeling complex NURF. Genes Dev 16 (24):3186-3198.

Baldi, C., J. Viviano, and R.E. Ellis, 2011 A bias caused by ectopic development produces sexually dimorphic sperm in nematodes. Curr Biol 21 (16):1416-1420.

Barker, D.M., 1994 Copulatory plugs and paternity assurance in the nematode Caenorhabditis elegans, pp. 147-156 in Animal Behaviour. Elsevier.

Barrière, A., and M.-A. Félix, 2005 High local genetic diversity and low outcrossing rate in Caenorhabditis elegans natural populations. Curr Biol 15 (13):1176-1184.

Barrière, A., and M.-A. Félix, 2007 Temporal dynamics and linkage disequilibrium in natural Caenorhabditis elegans populations, pp. 999-1011 in Genetics.

Bates, D., M. Mächler, B. Bolker, and S. Walker, 2015 Fitting Linear Mixed-Effects Models Using lme4. Journal of Statistical Software 67.

Birkhead, T.R., D.J. Hosken, and S. Pitnick, 2009 Sperm Biology: An Evolutionary Perspective. Boston: Academic Press (Elsevier).

Birkhead, T.R., and A.P. Moller, 1998 Sperm Competition and Sexual Selection. New York: Academic Press.

Bonduriansky, R., and S.F. Chenoweth, 2009 Intralocus sexual conflict. Trends Ecol Evol 24 (5):280-288.

Calhim, S., S. Immler, and T.R. Birkhead, 2007 Postcopulatory sexual selection is associated with reduced variation in sperm morphology. PLOS ONE 2 (5):e413.

Chasnov, J.R., 2013 The evolutionary role of males in C. elegans., pp. e21146 in Worm.

Chasnov, J.R., and K.L. Chow, 2002 Why are there males in the hermaphroditic species Caenorhabditis elegans? Genetics 160 (3):983-994.

Chasnov, J.R., W.K. So, C.M. Chan, and K.L. Chow, 2007 The species, sex, and stage specificity of a Caenorhabditis sex pheromone. Proc Natl Acad Sci U S A 104 (16):6730-6735.

Chen, X., Y. Shen, and R.E. Ellis, 2014 Dependence of the sperm/oocyte decision on the nucleosome remodeling factor complex was acquired during recent Caenorhabditis briggsae evolution. Mol Biol Evol 31 (10):2573-2585.

Chu, D.S., and D.C. Shakes, 2013 Spermatogenesis. Adv Exp Med Biol 757:171-203.

Cook, D.E., S. Zdraljevic, J.P. Roberts, and E.C. Andersen, 2017 CeNDR, the Caenorhabditis elegans natural diversity resource. Nucleic Acids Res 45 (D1):D650-d657.

Cook, D.E., S. Zdraljevic, R.E. Tanny, B. Seo, D.D. Riccardi et al., 2016 The Genetic Basis of Natural Variation in Caenorhabditis elegans Telomere Length. Genetics 204 (1):371-383.

Craig, H.L., J. Wirtz, S. Bamps, C.T. Dolphin, and I.A. Hope, 2013 The significance of alternative transcripts for Caenorhabditis elegans transcription factor genes, based on expression pattern analysis. BMC Genomics 14:249.

Cutter, A.D., 2004 Sperm-limited fecundity in nematodes: how many sperm are enough?, pp. 651-655 in Evolution.

Demarco, R.S., A.H. Eikenes, K. Haglund, and D.L. Jones, 2014 Investigating spermatogenesis in Drosophila melanogaster. Methods 68 (1):218-227.

Ellis, R.E., and G.M. Stanfield, 2014 The regulation of spermatogenesis and sperm function in nematodes. Semin Cell Dev Biol 29:17-30. 
Endelmann, J.B., 2011 Ridge Regression and Other Kernels for Genomic Selection with R Package rrBLUP. The Plant Genome 4:250-255.

Evans, K.S., Y. Zhao, S.C. Brady, L. Long, P.T. McGrath et al., 2017 Correlations of Genotype with Climate Parameters Suggest Caenorhabditis elegans Niche Adaptations. G3 (Bethesda) 7 (1):289-298.

Farhadifar, R., Charles F. Baer, A.-C. Valfort, Erik C. Andersen, T. Müller-Reichert et al., 2015 Scaling, selection, and evolutionary dynamics of the mitotic spindle. Curr Biol 25 (6):732-740.

Félix, M.-A., and C. Braendle, 2010 The natural history of Caenorhabditis elegans. Curr Biol 20 (22):R965-969.

Feng, H., H.L. Craig, and I.A. Hope, 2012 Expression pattern analysis of regulatory transcription factors in Caenorhabditis elegans. Methods Mol Biol 786:21-50.

Fitzpatrick, J.L., and B. Baer, 2011 Polyandry reduces sperm length variation in social insects. Evolution 65 (10):3006-3012.

Garcia, L.R., 2014 Regulation of sensory motor circuits used in C. elegans male intromission behavior. Semin Cell Dev Biol 33:42-49.

Geldziler, B.D., M.R. Marcello, D.C. Shakes, and A. Singson, 2011 The genetics and cell biology of fertilization. Methods Cell Biol 106:343-375.

Ghosh, R., E.C. Andersen, J.A. Shapiro, J.P. Gerke, and L. Kruglyak, 2012 Natural variation in a chloride channel subunit confers avermectin resistance in C. elegans, pp. 574-578 in Science.

Gomendio, M., J. Martin-Coello, C. Crespo, C. Magana, and E.R.S. Roldan, 2006 Sperm competition enhances functional capacity of mammalian spermatozoa. Proceedings of the National Academy of Sciences USA 103 (41):15113-15117.

Hansen, J.M., D.R. Chavez, and G.M. Stanfield, 2015 COMP-1 promotes competitive advantage of nematode sperm. Elife 4.

Hodgkin, J., and T. Barnes, 1991 More is not better: brood size and population growth in a self-fertilizing nematode, pp. 19-24 in Proceedings: Biological Sciences.

Hodgkin, J., and T. Doniach, 1997 Natural variation and copulatory plug formation in Caenorhabditis elegans, pp. 149-164 in Genetics.

Immler, S., S. Calhim, and T.R. Birkhead, 2008 Increased postcopulatory sexual selection reduces the intramale variation in sperm design. Evolution 62 (6):1538-1543.

Joly, D., A. Korol, and E. Nevo, 2004 Sperm size evolution in Drosophila: inter- and intraspecific analysis., pp. 233-244 in Genetica.

Jovelin, R., B.C. Ajie, and P.C. Phillips, 2003 Molecular evolution and quantitative variation for chemosensory behaviour in the nematode genus Caenorhabditis. Mol Ecol 12 (5):1325-1337.

Kamath, R., A. Fraser, Y. Dong, G. Poulin, R. Durbin et al., 2003 Systematic functional analysis of the Caenorhabditis elegans genome using RNAi, pp. 231-237 in Nature.

Kang, H.M., N.A. Zaitlen, C.M. Wade, A. Kirby, D. Heckerman et al., 2008 Efficient control of population structure in model organism association mapping. Genetics 178 (3):1709-1723.

Kleemann, G., and A. Basolo, 2007 Facultative decrease in mating resistance in hermaphroditic Caenorhabditis elegans with self-sperm depletion, pp. 1339-1347 in Animal Behaviour.

Kleven, O., T. Laskemoen, F. Fossøy, R.J. Robertson, and J.T. Lifjeld, 2008 Intraspecific variation in sperm length is negatively related to sperm competition in passerine birds, pp. 494-499 in Evolution. 
Kwon, S.Y., H. Xiao, C. Wu, and P. Badenhorst, 2009 Alternative splicing of NURF301 generates distinct NURF chromatin remodeling complexes with altered modified histone binding specificities. PLoS Genet 5 (7):e1000574.

L'Hernault, S.W., 2006 Spermatogenesis. WormBook:1-14.

LaMunyon, C.W., and S. Ward, 1995 Sperm precedence in a hermaphroditic nematode (Caenorhabditis elegans) is due to competitive superiority of male sperm. Experientia $51(8): 817-823$.

LaMunyon, C.W., and S. Ward, 1998 Larger sperm outcompete smaller sperm in the nematode Caenorhabditis elegans. Proceedings of the Royal Society of London Series B - Biological Sciences 265:1997-2002.

LaMunyon, C.W., and S. Ward, 1999 Evolution of sperm size in nematodes: Sperm competition favours larger sperm. Proceedings of the Royal Society of London Series B - Biological Sciences 266:263-267.

LaMunyon, C.W., and S. Ward, 2002 Evolution of larger sperm in response to experimentally increased sperm competition in Caenorhabditis elegans. Proceedings of the Royal Society of London Series B - Biological Sciences 269 (1496):1125-1128.

Large, E.E., R. Padmanabhan, K.L. Watkins, R.F. Campbell, W. Xu et al., 2017 Modeling of a negative feedback mechanism explains antagonistic pleiotropy in reproduction in domesticated Caenorhabditis elegans strains. PLoS Genet 13 (5):e1006769.

Large, E.E., W. Xu, Y. Zhao, S.C. Brady, L. Long et al., 2016 Selection on a Subunit of the NURF Chromatin Remodeler Modifies Life History Traits in a Domesticated Strain of Caenorhabditis elegans. PLoS Genet 12 (7):e1006219.

Li, H., 2011 A statistical framework for SNP calling, mutation discovery, association mapping and population genetical parameter estimation from sequencing data. Bioinformatics 27 (21):2987-2993.

Lipton, J., G. Kleemann, R. Ghosh, R. Lints, and S. Emmons, 2004 Mate searching in Caenorhabditis elegans: a genetic model for sex drive in a simple invertebrate, $\mathrm{pp}$. 7427 in Journal of Neuroscience.

Long, A., S. Mullaney, T. Mackay, and C. Langley, 1996 Genetic interactions between naturally occurring alleles at quantitative trait loci and mutant alleles at candidate loci affecting bristle number in Drosophila melanogaster. Genetics 144:1497-1510.

McGrath, P.T., M.V. Rockman, M. Zimmer, H. Jang, E.Z. Macosko et al., 2009 Quantitative mapping of a digenic behavioral trait implicates globin variation in C. elegans sensory behaviors. Neuron 61 (5):692-699.

McGrath, P.T., Y. Xu, M. Ailion, J.L. Garrison, R.A. Butcher et al., 2011 Parallel evolution of domesticated Caenorhabditis species targets pheromone receptor genes. Nature 477 (7364):321-325.

Miller, G.T., W.T. Starmer, and S. Pitnick, 2003 Quantitative genetic analysis of amongpopulation variation in sperm and female sperm-storage organ length in Drosophila mojavensis. Genet Res 81 (3):213-220.

Morran, L.T., B.J. Cappy, J.L. Anderson, and P.C. Phillips, 2009 Sexual partners for the stressed: facultative outcrossing in the self-fertilizing nematode Caenorhabditis elegans. Evolution 63 (6):1473-1482.

Morrow, E.H., and M. Gage, 2001a Artificial selection and heritability of sperm length in Gryllus bimaculatus in Heredity.

Morrow, E.H., and M.J.G. Gage, 2001b Consistent significant variation between individual males in spermatozoal morphometry. Journal of Zoology 254 (02):147-153.

Murray, R.L., J.L. Kozlowska, and A.D. Cutter, 2011 Heritable determinants of male fertilization success in the nematode Caenorhabditis elegans. BMC Evol Biol 11:99. 
Nelson, G.A., and S. Ward, 1980 Vesicle fusion, pseudopod extension and amoeboid motility are induced in nematode spermatids by the ionophore monensin. Cell 19:457-464.

Noble, L.M., A.S. Chang, D. McNelis, M. Kramer, M. Yen et al., 2015 Natural Variation in plep-1 Causes Male-Male Copulatory Behavior in C. elegans., pp. 2730-2737 in Curr Biol.

Palopoli, M.F., C. Peden, C. Woo, K. Akiha, M. Ary et al., 2015 Natural and experimental evolution of sexual conflict within Caenorhabditis nematodes. BMC Evol Biol 15:93.

Palopoli, M.F., M.V. Rockman, A. Tinmaung, C. Ramsay, S. Curwen et al., 2008 Molecular basis of the copulatory plug polymorphism in Caenorhabditis elegans. Nature 454 (7207):1019-1022.

Parker, G.A., and M.E. Begon, 1993 Sperm competition games: sperm size and number under gametic control. Proceedings of the Royal Society of London. Series B: Biological Sciences 253 (1338):255-262.

Pitnick, S., D.J. Hosken, and T.R. Birkhead, 2009 Sperm morphological diversity, pp. 69-149 in Sperm Biology: An Evolutionary Perspective, edited by T.R. Birkhead, D.J. Hosken and S. Pitnick. Academic Press (Elsevier), Boston.

Poullet, N., A. Vielle, C. Gimond, S. Carvalho, H. Teotonio et al., 2016 Complex heterochrony underlies the evolution of Caenorhabditis elegans hermaphrodite sex allocation. Evolution 70 (10):2357-2369.

Poullet, N., A. Vielle, C. Gimond, C. Ferrari, and C. Braendle, 2015 Evolutionarily divergent thermal sensitivity of germline development and fertility in hermaphroditic Caenorhabditis nematodes. Evol Dev 17 (6):380-397.

Price, A.L., G.V. Kryukov, P.I. de Bakker, S.M. Purcell, J. Staples et al., 2010 Pooled association tests for rare variants in exon-resequencing studies. Am J Hum Genet 86 (6):832-838.

Ramm, S.A., L. Schärer, J. Ehmcke, and J. Wistuba, 2014 Sperm competition and the evolution of spermatogenesis. Molecular Human Reproduction 20 (12):1169-1179.

Reece-Hoyes, J.S., J. Shingles, D. Dupuy, C.A. Grove, A.J. Walhout et al., 2007 Insight into transcription factor gene duplication from Caenorhabditis elegans Promoteromedriven expression patterns. BMC Genomics 8:27.

Ruthenburg, A.J., H. Li, T.A. Milne, S. Dewell, R.K. McGinty et al., 2011 Recognition of a mononucleosomal histone modification pattern by BPTF via multivalent interactions. Cell 145 (5):692-706.

Schedl, T., and J. Kimble, 1988 fog-2, a germ-line-specific sex determination gene required for hermaphrodite spermatogenesis in Caenorhabditis elegans. Genetics 119 (1):43-61.

Shakes, D.C., J.-C. Wu, P.L. Sadler, K. Laprade, L.L. Moore et al., 2009 Spermatogenesisspecific features of the meiotic program in Caenorhabditis elegans. PLoS Genetics 5 (8):e1000611.

Sherlekar, A.L., and R. Lints, 2014 Nematode Tango Milonguero - the C. elegans male's search for the hermaphrodite vulva. Semin Cell Dev Biol 33:34-41.

Simmons, L.W., and A.J. Moore, 2009 Evolutionary quantitative genetics of sperm, pp. 69149 in Sperm Biology: An Evolutionary Perspective, edited by T.R. Birkhead, D.J. Hosken and S. Pitnick. Academic Press (Elsevier), Boston.

Sivasundar, A., and J. Hey, 2005 Sampling from natural populations with RNAI reveals high outcrossing and population structure in Caenorhabditis elegans. Curr Biol 15 (17):1598-1602.

Smith, R.L., 1984 Sperm Competition and the Evolution of Animal Mating Systems. Orlando: Academic Press.

Snook, R.R., 2005 Sperm in competition: not playing by the numbers., pp. 46-53 in Trends Ecol Evol (Amst). 
Sterken, M.G., L.B. Snoek, J.E. Kammenga, and E.C. Andersen, 2015 The laboratory domestication of Caenorhabditis elegans, pp. 1-8 in Trends Genet. Elsevier Ltd.

Stiernagle, T., 2006 Maintenance of C. elegans. WormBook:1-11.

Teotonio, H., D. Manoel, and P.C. Phillips, 2006 Genetic variation for outcrossing among Caenorhabditis elegans isolates. Evolution 60 (6):1300-1305.

Thomas, C.G., G.C. Woodruff, and E.S. Haag, 2012 Causes and consequences of the evolution of reproductive mode in Caenorhabditis nematodes. Trends Genet 28 (5):213-220.

Timmons, L., and A. Fire, 1998 Specific interference by ingested dsRNA. Nature 395 (6705):854.

Ting, J.J., G.C. Woodruff, G. Leung, N.-R. Shin, A.D. Cutter et al., 2014 Intense spermmediated sexual conflict promotes gametic isolation in Caenorhabditis nematodes. PLoS Biology 12:e1001915.

Tsukiyama, T., C. Daniel, J. Tamkun, and C. Wu, 1995 ISWI, a member of the SWI2/SNF2 ATPase family, encodes the $140 \mathrm{kDa}$ subunit of the nucleosome remodeling factor. Cell 83 (6):1021-1026.

Vielle, A., N. Callemeyn-Torre, C. Gimond, N. Poullet, J.C. Gray et al., 2016 Convergent evolution of sperm gigantism and the developmental origins of sperm size variability in Caenorhabditis nematodes. Evolution 70 (11):2485-2503.

Ward, P.I., 1998 Intraspecific variation in sperm size characters. Heredity 80 ( Pt 6):655-659.

Ward, S., Y. Argon, and G.A. Nelson, 1981 Sperm morphogenesis in wild-type and fertilization-defective mutants of Caenorhabditis elegans Journal of Cell Biology 91:26-44.

Ward, S., and J.S. Carrel, 1979 Fertilization and sperm competition in the nematode Caenorhabditis elegans. Dev Biol 73 (2):304-321.

Wegewitz, V., H. Schulenburg, and A. Streit, 2008 Experimental insight into the proximate causes of male persistence variation among two strains of the androdioecious Caenorhabditis elegans (Nematoda), pp. 12 in BMC Ecol.

Wysocka, J., T. Swigut, H. Xiao, T.A. Milne, S.Y. Kwon et al., 2006 A PHD finger of NURF couples histone H3 lysine 4 trimethylation with chromatin remodelling. Nature 442 (7098):86-90.

Yin, D., E.M. Schwarz, C.G. Thomas, R.L. Felde, I.F. Korf et al., 2018 Rapid genome shrinkage in a self-fertile nematode reveals sperm competition proteins. Science 359 (6371):55-61.

Zdraljevic, S., C. Strand, H.S. Seidel, D.E. Cook, J.G. Doench et al., 2017 Natural variation in a single amino acid substitution underlies physiological responses to topoisomerase II poisons. PLoS Genet 13 (7):e1006891.

Zhan, X., Y. Hu, B. Li, G.R. Abecasis, and D.J. Liu, 2016 RVTESTS: an efficient and comprehensive tool for rare variant association analysis using sequence data. Bioinformatics 32 (9):1423-1426. 


\section{SUPPORTING INFORMATION}

Evolution of sperm competition: Natural variation and genetic determinants of Caenorhabditis elegans sperm size by Gimond et al.

\section{CONTENTS}

\section{Supporting Files}

File S1. Raw data in Excel format. Numbers below refer to different worksheets in Excel file

\section{Supporting Figures}

Figure S1 Effect of nurf-1 RNAi on male sperm size of $C$. elegans

Figure S2 Hermaphrodite sperm size of isw-1(n3297) compared to N2 wild-type and nurf-1(n4295)

Figure S3 Effect of nurf-1 RNAi on male sperm size of the gonochoristic species, $C$. plicata

\section{Supporting Tables}

Table S1. List of Caenorhabditis strains used in this study.

Table S2. Male sperm size variation in 97 C. elegans strains.

Table S3. Mean sperm size and plugging phenotype of examined C. elegans strains.

Table S4. Strain variation in hermaphrodite sperm size

Table S5. Strain variation in hermaphrodite sperm production

Table S6. Strain and sex differences in male and hermaphrodite body size 


\section{Supporting Files}

File S1. Raw data in Excel format. Numbers below refer to different worksheets in Excel file

1. Male sperm size of 97 C. elegans strains (measurements in microns)

(Figure 1)

2. Strain variation in male mating ability and fertility (Figures $3 A$ and $3 C$ )

3. Strain variation in ejaculate size (sperm number) (Figure 3E)

4. Strain variation in male fertilization success when competing with hermaphrodite self-sperm (Figure 4A)

5. Strain variation in male-male competitive ability (Figures $4 \mathrm{C}$ and $4 \mathrm{E}$ )

6. Strain variation in hermaphrodite sperm size (Figure 5A)

7. Strain variation in hermaphrodite sperm production (Figure $5 \mathrm{C}$ )

8. Strain variation in body size (Figure 6)

9. Male and hermaphrodite sperm size of strains N2, LSJ1, LSJ2 and CC1 (Figure 7)

10. Male and hermaphrodite sperm size of strains CX12311, CX13248, N2, and nurf-1(n4295) (Figure 8A-D)

11. Male sperm size measurements resulting from quantitative complementation tests using strains N2, LSJ1 and nurf-1(n4295) (Figure 8E) 12. Male sperm size of NURF complex mutants (Figure 9B)

13. Hermaphrodite sperm size of isw-1(n3297) versus N2 (Figure S2)

14. Male primary spermatocyte size of isw-1(n3297) versus N2 (Figure 9E)

15. Variation in body size of N2, LSJ1, nurf-1(n4295) and isw-1(n3294) (Figure 10)

16. Effect of nurf-1 RNAi on male sperm size of $C$. elegans (Figure S1) 17. Effect of nurf-1 RNAi on male sperm size of $C$. plicata (Figure S3) 


\section{Supporting Figures}

\section{Figure S1}

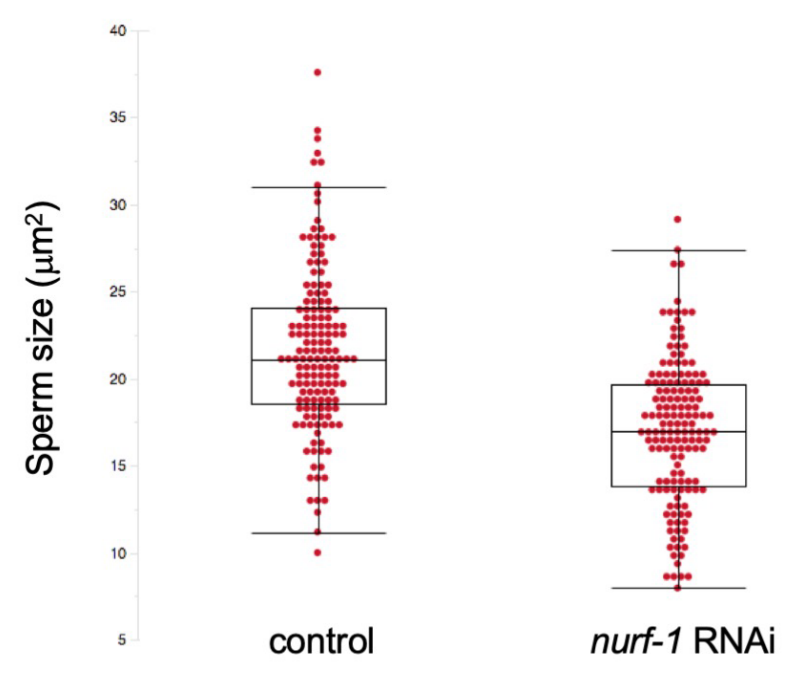

Figure S1 Effect of nurf-1 RNAi on male sperm size of C. elegans. nurf-1 RNAi significantly reduces male sperm size of the reference strain N2 (ANOVA, effect treatment: $F_{1,280}=90.91$, $\mathrm{P}<0.0001$; effect individual(treatment): $\mathrm{F}_{18,280}=3.07, \mathrm{P}<0.0001$ ). Fifteen spermatids from each of 10 individuals were measured per treatment $(\mathrm{N}=150)$. 


\section{Figure S2}

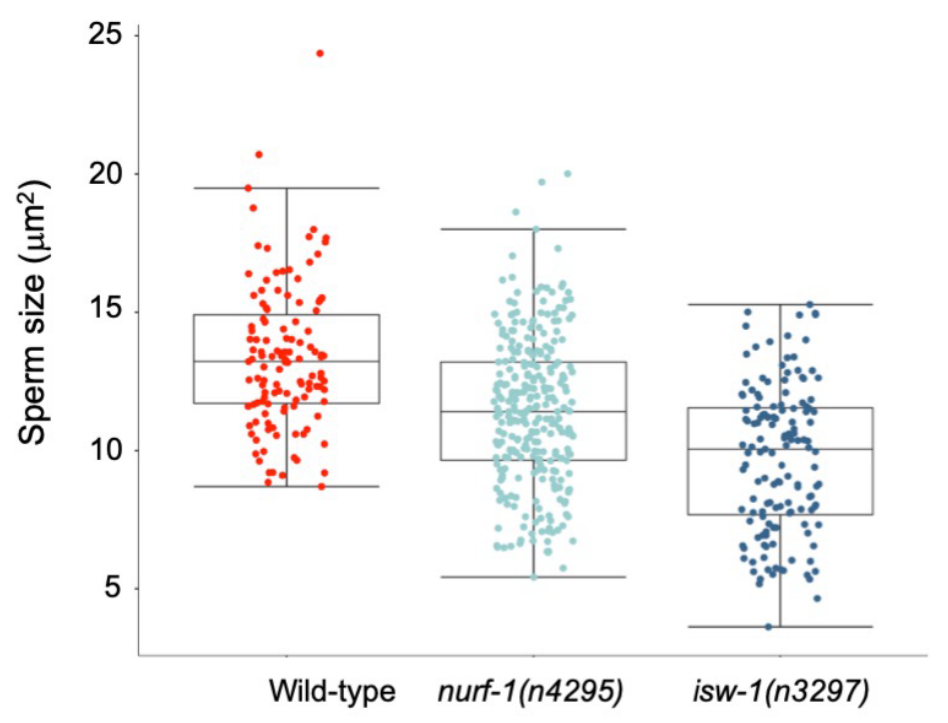

Figure S2 Hermaphrodite sperm size of isw-1(n3297) compared to N2 wild-type and nurf1(n4295). Hermaphrodite sperm size of isw-1(n3297) is significantly reduced compared to the N2 wild-type (ANOVA, effect strain: $F_{1,280}=90.91, P<0.0001$; effect individual(strain): $F_{18,280}=3.07$, $\mathrm{P}<0.0001)$. 


\section{Figure S3}

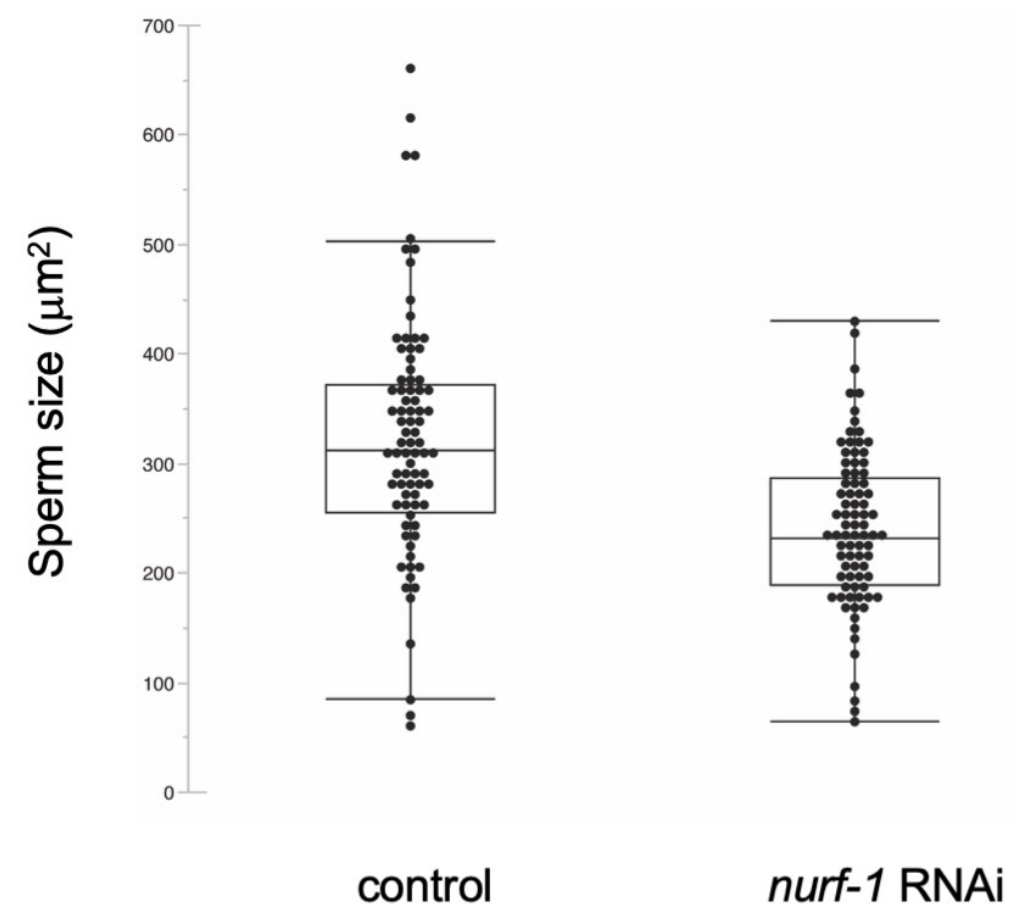

Figure S3 Effect of nurf-1 RNAi on male sperm size of the gonochoristic species, C. plicata. nurf-1 (C. elegans) RNAi significantly reduces male sperm size of the strain SB355 (ANOVA, effect treatment: $F_{1,163}=27.56, P<0.0001$; effect individual(treatment): $F_{10,163}=8.10, P<0.0001$ ). 8-15 spermatids from each of 7 individuals were measured per treatment. 


\section{Supporting Tables}

Table S1. List of Caenorhabditis strains used in this study. For additional strain information, see CeNDR: https://www.elegansvariation.org)

\begin{tabular}{|c|c|c|}
\hline STRAIN & ORIGIN & $\begin{array}{c}\text { DATE OF } \\
\text { ISOLATION }\end{array}$ \\
\hline $\mathrm{AB1}$ & Adelaide, Australia & 1983 \\
\hline AB4 & Adelaide, Australia & 1983 \\
\hline CB4851 & Bergerac, France & pre-1949 \\
\hline CB4852 & Unknown & pre-1966 \\
\hline CB4853 & Altadena, USA & $1974 / 05$ \\
\hline CB4854 & Altadena, USA & $1974 / 05$ \\
\hline CB4856 & Oahu, Hawaii & $1972 / 08$ \\
\hline CB4857 & Claremont, USA & $1972 / 11$ \\
\hline CB4858 & Pasadena, USA & 1973 \\
\hline CB4932 & Taunton, United Kingdom & pre-1991 \\
\hline CX11262 & Los Angeles, USA & $2003 / 09$ \\
\hline CX11264 & Los Angeles, USA & $2003 / 09$ \\
\hline CX11271 & Pasadena, USA & $2003 / 09$ \\
\hline CX11276 & Los Angeles, USA & $2003 / 09$ \\
\hline CX11285 & Los Angeles, USA & $2003 / 09$ \\
\hline CX11292 & Los Angeles, USA & $2004 / 02$ \\
\hline CX11307 & Los Angeles, USA & $2003 / 09$ \\
\hline CX11314 & Los Angeles, USA & $2003 / 09$ \\
\hline CX11315 & Los Angeles, USA & $2003 / 09$ \\
\hline DL200 & Addis Ababa, Ethiopia & $2007 / 12$ \\
\hline DL226 & Corvallis, USA & 2007 \\
\hline DL238 & Manuka, Hawaii & $2008 / 07 / 15$ \\
\hline ED3005 & Edinburgh, United Kingdom & $2004 / 10 / 25$ \\
\hline ED3011 & Edinburgh, United Kingdom & $2004 / 11 / 26$ \\
\hline ED3012 & Edinburgh, United Kingdom & $2004 / 11 / 26$ \\
\hline ED3017 & Edinburgh, United Kingdom & $2004 / 12 / 03$ \\
\hline ED3040 & Johannesburg, South Africa & $2006 / 03$ \\
\hline ED3046 & Ceres, South Africa & $2006 / 03$ \\
\hline ED3048 & Ceres, South Africa & $2006 / 03$ \\
\hline ED3049 & Ceres, South Africa & $2006 / 03$ \\
\hline ED3052 & Ceres, South Africa & $2006 / 03$ \\
\hline ED3073 & Limuru, Kenya & $2006 / 03$ \\
\hline ED3077 & Nairobi, Kenya & $2006 / 03$ \\
\hline EG4347 & Eugene, USA & $2006 / 10$ \\
\hline EG4349 & Salt Lake City, USA & $2006 / 10$ \\
\hline EG4724 & Amares, Portugal & $2007 / 03$ \\
\hline EG4725 & Amares, Portugal & $2007 / 03$ \\
\hline EG4946 & Salt Lake City, USA & $2007 / 09 / 27$ \\
\hline JT11398 & Lake Forest Park, USA & $2003 / 12$ \\
\hline JU1088 & Kakegawa, Japan & $2007 / 03 / 14$ \\
\hline JU1172 & Concepcion, Chile & $2007 / 04$ \\
\hline JU1200 & Dundonald, United Kingdom & $2007 / 08 / 01$ \\
\hline JU1212 & Primel Trégastel, France & $2007 / 09 / 24$ \\
\hline JU1213 & Primel Trégastel, France & $2007 / 09 / 24$ \\
\hline JU1242 & Santeuil, France & $2007 / 10 / 14$ \\
\hline JU1246 & Santeuil, France & $2007 / 10 / 14$ \\
\hline JU1395 & Saut-aux-Loups, France & $2008 / 03 / 01$ \\
\hline JU1400 & Sevilla, Spain & $2008 / 03$ \\
\hline JU1409 & Carmona, Spain & $2008 / 03 / 31$ \\
\hline JU1440 & Barcelona, Spain & $2008 / 06 / 09$ \\
\hline JU1491 & Le Blanc, France & $2008 / 08 / 17$ \\
\hline JU1530 & Orsay, France & $2008 / 09 / 09$ \\
\hline JU1568 & Ivry-sur-Seine, France & $2008 / 10 / 05$ \\
\hline JU1580 & Orsay, France & $2008 / 10 / 06$ \\
\hline JU1581 & Orsay, France & $2008 / 10 / 23$ \\
\hline JU1586 & Le Blanc, France & $2008 / 11 / 03$ \\
\hline JU1652 & Montevideo, Uruguay & 2009 \\
\hline JU1896 & Athens, Greece & $2010 / 01 / 02$ \\
\hline JU258 & Ribeiro Frio, Madeira & $2001 / 10$ \\
\hline
\end{tabular}


bioRxiv preprint doi: https://doi.org/10.1101/501486; this version posted December 19, 2018. The copyright holder for this preprint (which was not certified by peer review) is the author/funder. All rights reserved. No reuse allowed without permission.

\begin{tabular}{|c|c|c|}
\hline JU310 & Le Blanc, France & $2002 / 08 / 25$ \\
\hline JU311 & Merlet, France & $2002 / 09 / 08$ \\
\hline JU323 & Merlet, France & $2002 / 09 / 08$ \\
\hline JU346 & Merlet, France & $2002 / 09 / 08$ \\
\hline JU360 & Franconville, France & 2002/09/02 \\
\hline JU363 & Franconville, France & $2002 / 09 / 16$ \\
\hline JU367 & Franconville, France & $2002 / 09 / 16$ \\
\hline JU393 & Hermanville, France & $2002 / 09$ \\
\hline JU394 & Hermanville, France & $2002 / 09$ \\
\hline JU397 & Hermanville, France & $2002 / 09$ \\
\hline $\mathrm{JU} 406$ & Hermanville, France & $2002 / 12 / 30$ \\
\hline JU440 & Beauchêne, France & 2003/09/12 \\
\hline JU561 & Sainte-Barbe, France & 2004/10/03 \\
\hline JU642 & Le Perreux-sur-Marne, France & 2004/12/14 \\
\hline JU751 & Le Perreux-sur-Marne, France & 2005/06/08 \\
\hline JU774 & Carcavelos, Portugal & $2005 / 07 / 10$ \\
\hline JU775 & Lisbon, Portugal & $2005 / 07 / 10$ \\
\hline JU778 & Lisbon, Portugal & $2005 / 07 / 10$ \\
\hline JU782 & Lisbon, Portugal & $2005 / 07 / 10$ \\
\hline JU792 & Fréchendets, France & $2005 / 08 / 31$ \\
\hline JU830 & Tübingen, Germany & $2005 / 09 / 28$ \\
\hline JU847 & Obernai, France & 2005/10/03 \\
\hline KR314 & Vancouver, Canada & $1984 / 05$ \\
\hline LKC34 & Unknown city, Madagascar & 2005/06/17 \\
\hline LSJ1 & Bristol, United Kingdom & 1951 \\
\hline $\mathrm{N} 2$ & Bristol, United Kingdom & 1951 \\
\hline MY1 & Lingen, Germany & $2002 / 07$ \\
\hline MY10 & Roxel, Germany & $2002 / 07$ \\
\hline MY16 & Mecklenbeck, Germany & $2002 / 07$ \\
\hline MY18 & Roxel, Germany & $2002 / 07$ \\
\hline MY23 & Roxel, Germany & $2002 / 07$ \\
\hline PB303 & NA & 1998/11/14 \\
\hline PB306 & $\mathrm{NA}$ & $1998 / 11 / 28$ \\
\hline PS2025 & Altadena, USA & Unknown \\
\hline PX179 & Eugene, USA & $2001 / 10 / 02$ \\
\hline QX1211 & San Francisco, USA & $2007 / 11 / 26$ \\
\hline QX1233 & Berkeley, USA & $2007 / 11 / 24$ \\
\hline RC301 & Freiburg, Germany & 1983 \\
\hline WN2002 & Wageningen, Netherlands & $2007 / 11 / 20$ \\
\hline
\end{tabular}


bioRxiv preprint doi: https://doi.org/10.1101/501486; this version posted December 19, 2018. The copyright holder for this preprint (which

was not certified by peer review) is the author/funder. All rights reserved. No reuse allowed without permission.

Table S2. Male sperm size variation (means of cross-sectional area, microns) and CV in $97 \mathrm{C}$. elegans strains.

\begin{tabular}{|c|c|c|c|c|c|c|}
\hline Strain & $\begin{array}{l}\text { Sperm Size } \\
\text { (mean) }\end{array}$ & SEM & $\begin{array}{l}\text { CV (within- } \\
\text { individual) }\end{array}$ & $\begin{array}{l}\text { CV (betwee- } \\
\text { individual) }\end{array}$ & $\begin{array}{l}\mathbf{N} \\
\text { individuals }\end{array}$ & $\begin{array}{l}\mathrm{N} \\
\text { sperm }\end{array}$ \\
\hline $\mathrm{AB} 1$ & 23.94 & 1.68 & 16.49 & 5.22 & 7 & 140 \\
\hline AB4 & 22.13 & 0.83 & 18.75 & 6.46 & 7 & 140 \\
\hline CB4852 & 21.44 & 1.91 & 17.55 & 5.68 & 7 & 140 \\
\hline CB4853 & 21.98 & 2.36 & 22.13 & 4.66 & 7 & 140 \\
\hline CB4854 & 25.07 & 1.73 & 21.09 & 4.05 & 7 & 140 \\
\hline CB4856 & 22.83 & 0.70 & 16.42 & 5.60 & 7 & 140 \\
\hline CB4857 & 22.89 & 2.05 & 20.67 & 4.35 & 7 & 140 \\
\hline CB4858 & 22.08 & 1.53 & 17.63 & 7.01 & 7 & 140 \\
\hline CB4932 & 21.06 & 0.88 & 15.65 & 7.69 & 7 & 140 \\
\hline CX11262 & 21.81 & 1.65 & 16.93 & 8.79 & 7 & 140 \\
\hline CX11264 & 20.85 & 1.29 & 16.33 & 4.91 & 7 & 140 \\
\hline CX11271 & 25.03 & 1.55 & 18.86 & 7.04 & 7 & 140 \\
\hline CX11276 & 21.56 & 1.24 & 18.82 & 6.23 & 7 & 140 \\
\hline CX11285 & 18.97 & 1.60 & 17.16 & 6.81 & 7 & 140 \\
\hline CX11292 & 21.40 & 1.51 & 19.59 & 7.48 & 7 & 140 \\
\hline CX11307 & 23.95 & 1.82 & 17.03 & 5.90 & 7 & 140 \\
\hline CX11314 & 20.76 & 1.83 & 18.55 & 6.06 & 7 & 140 \\
\hline CX11315 & 20.82 & 1.36 & 15.20 & 7.69 & 7 & 140 \\
\hline DL200 & 20.62 & 0.62 & 16.97 & 3.31 & 7 & 140 \\
\hline DL226 & 24.36 & 2.09 & 18.99 & 5.82 & 7 & 140 \\
\hline DL238 & 21.29 & 1.52 & 19.70 & 8.92 & 7 & 140 \\
\hline ED3005 & 24.36 & 1.47 & 24.36 & 5.44 & 7 & 140 \\
\hline ED3011 & 21.17 & 1.39 & 25.29 & 13.02 & 7 & 140 \\
\hline ED3012 & 22.72 & 1.05 & 16.88 & 6.27 & 7 & 140 \\
\hline ED3017 & 23.23 & 1.70 & 20.66 & 4.92 & 7 & 140 \\
\hline ED3040 & 22.45 & 1.14 & 20.66 & 9.96 & 7 & 140 \\
\hline ED3046 & 24.32 & 1.22 & 18.63 & 4.75 & 7 & 140 \\
\hline ED3048 & 20.73 & 2.29 & 19.68 & 6.12 & 7 & 140 \\
\hline ED3049 & 21.68 & 0.72 & 13.74 & 4.87 & 7 & 140 \\
\hline ED3052 & 21.97 & 1.91 & 16.82 & 7.09 & 7 & 140 \\
\hline ED3073 & 22.98 & 1.45 & 16.42 & 5.25 & 7 & 140 \\
\hline ED3077 & 22.63 & 0.79 & 20.15 & 6.86 & 7 & 140 \\
\hline EG4347 & 24.05 & 1.28 & 19.25 & 7.10 & 7 & 140 \\
\hline EG4349 & 21.49 & 2.00 & 18.71 & 5.43 & 7 & 140 \\
\hline EG4724 & 20.96 & 2.24 & 14.97 & 6.88 & 7 & 140 \\
\hline EG4725 & 21.08 & 1.24 & 18.07 & 8.55 & 7 & 140 \\
\hline EG4946 & 25.55 & 1.68 & 19.75 & 6.28 & 7 & 140 \\
\hline JT11398 & 22.17 & 1.80 & 15.50 & 5.90 & 7 & 140 \\
\hline JU258 & 20.79 & 1.46 & 15.15 & 5.98 & 7 & 140 \\
\hline JU310 & 24.36 & 1.23 & 19.54 & 5.79 & 7 & 140 \\
\hline JU311 & 24.06 & 2.07 & 20.87 & 13.30 & 7 & 140 \\
\hline JU323 & 23.80 & 2.55 & 22.45 & 6.77 & 7 & 140 \\
\hline JU346 & 23.35 & 1.66 & 14.92 & 5.96 & 7 & 140 \\
\hline JU360 & 23.91 & 1.34 & 17.15 & 7.36 & 7 & 140 \\
\hline JU363 & 24.90 & 1.38 & 19.22 & 5.31 & 7 & 140 \\
\hline JU367 & 23.37 & 0.94 & 14.16 & 5.77 & 7 & 140 \\
\hline JU393 & 26.70 & 0.92 & 18.15 & 10.12 & 7 & 140 \\
\hline JU394 & 24.16 & 1.39 & 16.32 & 4.12 & 7 & 140 \\
\hline JU397 & 23.12 & 0.95 & 12.10 & 6.74 & 6 & 120 \\
\hline JU406 & 21.36 & 1.96 & 17.72 & 7.32 & 7 & 140 \\
\hline JU440 & 23.22 & 1.01 & 17.77 & 6.27 & 7 & 140 \\
\hline JU561 & 15.92 & 2.06 & 19.17 & 12.52 & 7 & 140 \\
\hline JU642 & 21.21 & 1.55 & 13.97 & 11.23 & 7 & 140 \\
\hline JU751 & 21.94 & 2.41 & 18.75 & 9.02 & 7 & 140 \\
\hline JU774 & 22.10 & 1.68 & 21.79 & 10.10 & 7 & 140 \\
\hline JU775 & 22.48 & 1.89 & 21.71 & 10.49 & 7 & 140 \\
\hline JU778 & 20.37 & 1.46 & 22.84 & 8.98 & 7 & 140 \\
\hline JU782 & 26.29 & 2.68 & 23.26 & 3.23 & 7 & 140 \\
\hline JU792 & 20.59 & 1.40 & 14.52 & 7.78 & 7 & 140 \\
\hline JU830 & 24.16 & 1.06 & 15.54 & 3.07 & 7 & 140 \\
\hline JU847 & 22.06 & 1.73 & 17.73 & 5.16 & 7 & 140 \\
\hline JU1088 & 23.41 & 1.19 & 14.26 & 8.82 & 7 & 140 \\
\hline JU1172 & 22.57 & 2.48 & 20.17 & 5.61 & 7 & 140 \\
\hline JU1200 & 22.00 & 1.37 & 18.35 & 3.85 & 7 & 140 \\
\hline
\end{tabular}


bioRxiv preprint doi: https://doi.org/10.1101/501486; this version posted December 19, 2018. The copyright holder for this preprint (which

was not certified by peer review) is the author/funder. All rights reserved. No reuse allowed without permission.

\begin{tabular}{|c|c|c|c|c|c|c|}
\hline JU1212 & 21.61 & 1.51 & 18.11 & 3.14 & 7 & 140 \\
\hline JU1213 & 23.80 & 1.44 & 24.69 & 21.07 & 7 & 140 \\
\hline JU1242 & 19.99 & 1.00 & 15.13 & 12.12 & 7 & 140 \\
\hline JU1246 & 21.40 & 2.59 & 19.01 & 7.33 & 7 & 140 \\
\hline JU1395 & 22.77 & 1.04 & 17.56 & 2.38 & 7 & 140 \\
\hline JU1400 & 19.56 & 1.91 & 18.74 & 5.19 & 7 & 140 \\
\hline JU1409 & 24.64 & 0.91 & 13.12 & 5.23 & 7 & 140 \\
\hline JU1440 & 20.49 & 2.44 & 19.04 & 4.92 & 7 & 140 \\
\hline JU1491 & 21.70 & 1.71 & 21.68 & 3.81 & 7 & 140 \\
\hline JU1530 & 25.71 & 1.63 & 17.35 & 20.12 & 7 & 140 \\
\hline JU1568 & 22.09 & 1.91 & 16.93 & 6.42 & 7 & 140 \\
\hline JU1580 & 22.00 & 0.76 & 15.02 & 7.87 & 7 & 140 \\
\hline JU1581 & 20.41 & 1.22 & 13.76 & 3.65 & 7 & 140 \\
\hline JU1586 & 20.16 & 1.47 & 19.50 & 9.14 & 7 & 140 \\
\hline JU1652 & 21.95 & 1.59 & 17.23 & 6.97 & 7 & 140 \\
\hline JU1896 & 23.21 & 0.81 & 18.26 & 8.87 & 7 & 140 \\
\hline KR314 & 22.11 & 3.11 & 21.29 & 14.20 & 6 & 120 \\
\hline LKC34 & 24.68 & 1.06 & 18.99 & 5.75 & 7 & 140 \\
\hline LSJ1 & 15.25 & 0.82 & 16.09 & 9.08 & 7 & 140 \\
\hline MY1 & 20.01 & 1.56 & 16.72 & 4.62 & 7 & 140 \\
\hline MY10 & 20.84 & 1.54 & 20.29 & 9.43 & 7 & 140 \\
\hline MY16 & 22.46 & 1.79 & 16.80 & 1.66 & 7 & 140 \\
\hline MY18 & 22.16 & 1.12 & 20.60 & 10.50 & 7 & 140 \\
\hline MY23 & 20.09 & 1.65 & 16.80 & 7.44 & 7 & 140 \\
\hline $\mathrm{N} 2$ & 19.51 & 2.17 & 20.68 & 3.70 & 7 & 140 \\
\hline PB303 & 23.01 & 0.83 & 18.10 & 4.90 & 7 & 140 \\
\hline PB306 & 22.15 & 1.47 & 18.60 & 5.82 & 7 & 140 \\
\hline PS2025 & 22.40 & 1.48 & 19.73 & 7.99 & 7 & 140 \\
\hline PX179 & 22.41 & 1.81 & 21.73 & 7.20 & 7 & 140 \\
\hline QX1211 & 22.11 & 1.02 & 18.49 & 6.97 & 7 & 140 \\
\hline QX1233 & 23.57 & 2.81 & 23.73 & 19.28 & 7 & 140 \\
\hline RC301 & 21.86 & 0.95 & 16.92 & 6.65 & 7 & 140 \\
\hline WN2002 & 22.37 & 2.06 & 20.86 & 7.31 & 7 & 140 \\
\hline
\end{tabular}


bioRxiv preprint doi: https://doi.org/10.1101/501486; this version posted December 19, 2018. The copyright holder for this preprint (which was not certified by peer review) is the author/funder. All rights reserved. No reuse allowed without permission.

Table S3. Mean sperm size and plugging phenotype of examined C. elegans strains. Plugging phenotype data from Andersen et al. (2012) and Palopoli et al. (2015).

\begin{tabular}{|c|c|c|}
\hline Strain & Mean sperm size & Plugging $=1$, Non-plugging $=0$ \\
\hline $\mathrm{AB} 1$ & 23.5511 & 0 \\
\hline AB4 & 21.659 & 1 \\
\hline CB4852 & 21.0393 & 0 \\
\hline CB4853 & 21.356 & 1 \\
\hline CB4854 & 24.4207 & 1 \\
\hline CB4856 & 22.4802 & 1 \\
\hline CB4857 & 22.3462 & 0 \\
\hline CB4858 & 21.6617 & 1 \\
\hline CB4932 & 20.7466 & 0 \\
\hline CX11262 & 21.3944 & 1 \\
\hline CX11264 & 20.537 & 1 \\
\hline CX11271 & 24.5216 & 1 \\
\hline CX11276 & 21.1239 & 1 \\
\hline CX11285 & 18.6448 & 1 \\
\hline CX11292 & 20.9053 & 1 \\
\hline CX11307 & 23.5335 & 1 \\
\hline CX11314 & 20.3464 & 1 \\
\hline CX11315 & 20.5113 & 1 \\
\hline DL200 & 20.3174 & 1 \\
\hline DL226 & 23.8156 & 1 \\
\hline DL238 & 20.7834 & 1 \\
\hline ED3005 & 23.5608 & 1 \\
\hline ED3011 & 20.2955 & 1 \\
\hline ED3012 & 22.3289 & 0 \\
\hline ED3017 & 22.6823 & 1 \\
\hline ED3040 & 21.8398 & 1 \\
\hline ED3046 & 23.8324 & 1 \\
\hline ED3048 & 20.2543 & 1 \\
\hline ED3049 & 21.45 & 1 \\
\hline ED3052 & 21.5752 & 1 \\
\hline ED3073 & 22.6221 & 1 \\
\hline ED3077 & 22.0667 & 1 \\
\hline EG4347 & 23.5467 & 0 \\
\hline EG4349 & 21.0612 & 1 \\
\hline EG4724 & 20.6426 & 1 \\
\hline EG4725 & 20.631 & 1 \\
\hline EG4946 & 24.9506 & 0 \\
\hline JT11398 & 21.8455 & 0 \\
\hline JU1088 & 23.0792 & 1 \\
\hline JU1172 & 22.0359 & 1 \\
\hline JU1200 & 21.58 & 0 \\
\hline JU1212 & 21.2382 & 0 \\
\hline JU1213 & 22.6857 & 1 \\
\hline JU1242 & 19.6241 & 1 \\
\hline JU1246 & 20.9496 & 1 \\
\hline JU1395 & 22.3787 & 0 \\
\hline JU1400 & 19.1491 & 1 \\
\hline JU1409 & 24.3819 & 1 \\
\hline JU1440 & 20.0642 & 1 \\
\hline JU1491 & 21.1306 & 1 \\
\hline JU1530 & 24.8259 & 1 \\
\hline JU1568 & 21.6964 & 0 \\
\hline JU1580 & 21.6949 & 1 \\
\hline JU1581 & 20.1968 & 1 \\
\hline JU1586 & 19.6909 & 0 \\
\hline JU1652 & 21.5463 & 1 \\
\hline JU1896 & 22.7062 & 0 \\
\hline JU258 & 20.4938 & 1 \\
\hline JU310 & 23.8629 & 0 \\
\hline JU311 & 23.3055 & 0 \\
\hline JU323 & 23.1108 & 1 \\
\hline JU346 & 23.0378 & 1 \\
\hline JU360 & 23.4768 & 1 \\
\hline JU363 & 24.3861 & 1 \\
\hline
\end{tabular}


bioRxiv preprint doi: https://doi.org/10.1101/501486; this version posted December 19, 2018. The copyright holder for this preprint (which

was not certified by peer review) is the author/funder. All rights reserved. No reuse allowed without permission.

\begin{tabular}{|c|c|c|}
\hline JU367 & 23.1158 & 0 \\
\hline JU393 & 26.1593 & 1 \\
\hline JU394 & 23.8254 & 0 \\
\hline JU397 & 22.9068 & 1 \\
\hline JU406 & 20.9359 & 0 \\
\hline JU440 & 22.8045 & 0 \\
\hline JU561 & 15.4953 & 1 \\
\hline JU642 & 20.8737 & 1 \\
\hline JU751 & 21.4432 & 1 \\
\hline JU774 & 21.4306 & 1 \\
\hline JU775 & 21.8151 & 1 \\
\hline JU778 & 19.7247 & 1 \\
\hline JU782 & 25.4637 & 1 \\
\hline JU792 & 20.3049 & 1 \\
\hline JU830 & 23.8397 & 1 \\
\hline JU847 & 21.6807 & 1 \\
\hline KR314 & 21.366 & 1 \\
\hline LKC34 & 24.1826 & 1 \\
\hline LSJ1 & 15.0017 & 0 \\
\hline MY1 & 19.7027 & 1 \\
\hline MY10 & 20.3428 & 1 \\
\hline MY16 & 22.107 & 1 \\
\hline MY18 & 21.557 & 1 \\
\hline MY23 & 19.733 & 1 \\
\hline $\mathrm{N} 2$ & 19.0539 & 0 \\
\hline PB303 & 22.6011 & 1 \\
\hline PB306 & 21.7096 & 1 \\
\hline PS2025 & 21.8714 & 1 \\
\hline PX179 & 21.788 & 0 \\
\hline QX1211 & 21.6496 & 1 \\
\hline QX1233 & 22.4217 & 1 \\
\hline RC301 & 21.4845 & 1 \\
\hline WN2002 & 21.7914 & 0 \\
\hline
\end{tabular}


Table S4. Strain variation in hermaphrodite sperm size (and comparison to male sperm size) (crosssectional area in microns) (data shown in figure 5A).

\begin{tabular}{|c|c|c|c|c|c|}
\hline Strain & Sex & Mean sperm size & SEM & $\mathrm{N}$ individuals & N spermatids \\
\hline LSJ1 & Male & 15.25 & 0.23 & 7 & 140 \\
\hline LSJ1 & Hermaphrodite & 10.44 & 0.20 & 11 & 143 \\
\hline JU561 & Male & 15.91 & 0.31 & 7 & 140 \\
\hline JU561 & Hermaphrodite & 10.34 & 0.17 & 12 & 134 \\
\hline CX11285 & Male & 18.97 & 0.29 & 7 & 140 \\
\hline CX11285 & Hermaphrodite & 12.04 & 0.22 & 9 & 104 \\
\hline N2 & Male & 19.51 & 0.35 & 7 & 140 \\
\hline $\mathrm{N} 2$ & Hermaphrodite & 13.37 & 0.23 & 9 & 123 \\
\hline DL238 & Male & 21.29 & 0.39 & 7 & 140 \\
\hline DL238 & Hermaphrodite & 9.96 & 0.19 & 8 & 140 \\
\hline JU751 & Male & 21.94 & 0.38 & 7 & 140 \\
\hline JU751 & Hermaphrodite & 11.65 & 0.25 & 10 & 99 \\
\hline JU1200 & Male & 21.99 & 0.34 & 7 & 140 \\
\hline JU1200 & Hermaphrodite & 12.16 & 0.20 & 12 & 136 \\
\hline JU775 & Male & 22.48 & 0.46 & 7 & 140 \\
\hline JU775 & Hermaphrodite & 9.04 & 0.24 & 7 & 87 \\
\hline CB4856 & Male & 22.8 & 0.33 & 7 & 140 \\
\hline CB4856 & Hermaphrodite & 13.25 & 0.21 & 13 & 149 \\
\hline EG4946 & Male & 25.55 & 0.44 & 7 & 140 \\
\hline EG4946 & Hermaphrodite & 11.07 & 0.25 & 8 & 125 \\
\hline JU782 & Male & 26.29 & 0.53 & 7 & 140 \\
\hline JU782 & Hermaphrodite & 12.94 & 0.24 & 10 & 136 \\
\hline JU393 & Male & 26.70 & 0.45 & 7 & 140 \\
\hline JU393 & Hermaphrodite & 13.19 & 0.32 & 7 & 152 \\
\hline
\end{tabular}


Table S5. Strain variation in hermaphrodite sperm production (data in Figure 5C).

\begin{tabular}{|l|l|l|l|}
\hline Strain & $\begin{array}{l}\text { Mean sperm } \\
\text { number/spermatheca }\end{array}$ & SEM & $\mathbf{N}$ individuals \\
\hline CB4856 & 137.00 & 3.28 & 31 \\
\hline CX11285 & 146.65 & 3.98 & 20 \\
\hline EG4946 & 151.83 & 7.22 & 23 \\
\hline JU393 & 150.15 & 5.48 & 20 \\
\hline JU561 & 136.38 & 5.20 & 29 \\
\hline JU782 & 152.74 & 4.00 & 27 \\
\hline LSJ1 & 175.96 & 7.81 & 23 \\
\hline N2 & 145.00 & 5.28 & 12 \\
\hline
\end{tabular}


Table S6. Strain and sex differences in male and hermaphrodite body size (measures in microns). Strain and sex had significant effects on body length (ANOVA, effect sex: $F_{1,422}=2325.80, P<0.0001$; effect strain: $F_{10,422}=12.78, P<0.0001$; interaction sex $x$ strain: $F_{10,422}=23.57, P<0.0001$ ) and body width (ANOVA, effect sex: $F_{1,422}=1549,46, P<0.0001$; effect strain: $F_{10,422}=15.25, P<0.0001$; interaction sex $x$ strain: $F_{10,422}=19.47, P<0.0001$ ). (ANOVA analyses testing for the fixed effects of strain and sex on body length/width; all data log-transformed).

\begin{tabular}{|c|c|c|c|c|c|c|}
\hline Strain & Sex & Body length & SE length & $\begin{array}{l}\text { Body } \\
\text { width }\end{array}$ & $\begin{array}{l}\text { SE } \\
\text { width }\end{array}$ & $\begin{array}{l}\mathbf{N} \\
\text { individuals }\end{array}$ \\
\hline LSJ1 & $M$ & 716.5 & 7.9 & 40.9 & 0.6 & 24 \\
\hline LSJ1 & $\mathrm{H}$ & 998 & 11.2 & 62 & 0.9 & 23 \\
\hline JU561 & $\mathrm{M}$ & 732.9 & 8.1 & 43.1 & 0.4 & 19 \\
\hline JU561 & $\mathrm{H}$ & 1012 & 13.3 & 56 & 1.0 & 18 \\
\hline CX11285 & $\mathrm{M}$ & 722.2 & 12.5 & 40.2 & 0.8 & 19 \\
\hline CX11285 & $\mathrm{H}$ & 960 & 8.0 & 62 & 0.6 & 18 \\
\hline N2 & $M$ & 786.8 & 8.1 & 43.7 & 0.6 & 23 \\
\hline N2 & $\mathrm{H}$ & 1057 & 9.2 & 62 & 0.7 & 20 \\
\hline DL238 & $\mathrm{M}$ & 794.7 & 8.2 & 45.2 & 0.9 & 21 \\
\hline DL238 & $\mathrm{H}$ & 994 & 12.0 & 63 & 1.1 & 17 \\
\hline JU751 & $\mathrm{M}$ & 822.3 & 10.0 & 43.4 & 0.7 & 15 \\
\hline JU751 & $\mathrm{H}$ & 1120 & 13.8 & 63 & 1.2 & 17 \\
\hline JU1200 & $\mathrm{M}$ & 800.1 & 7.8 & 46.6 & 0.3 & 21 \\
\hline JU1200 & $\mathrm{H}$ & 1140 & 13.2 & 67 & 0.8 & 21 \\
\hline CB4856 & $\mathrm{M}$ & 747.8 & 7.9 & 43.6 & 0.4 & 21 \\
\hline CB4856 & $\mathrm{H}$ & 965 & 8.3 & 60 & 0.4 & 19 \\
\hline EG4946 & $M$ & 716.1 & 12.1 & 47.2 & 1.2 & 10 \\
\hline EG4946 & $\mathrm{H}$ & 974 & 9.1 & 65 & 1.1 & 16 \\
\hline JU782 & $\mathrm{M}$ & 819.0 & 5.8 & 48.4 & 0.8 & 24 \\
\hline JU782 & $\mathrm{H}$ & 972 & 10.7 & 62 & 0.9 & 15 \\
\hline JU393 & $\mathrm{M}$ & 764.0 & 6.9 & 47.2 & 0.7 & 17 \\
\hline JU393 & $\mathrm{H}$ & 1018 & 9.0 & 62 & 0.7 & 29 \\
\hline
\end{tabular}

